\title{
New Polarization Basis for Polarimetric Phased Array Weather Radar: Theory and Polarimetric Variables Measurement
}

\author{
Jian Dong, Qingfu Liu, and Xuesong Wang \\ School of Electronic Science and Engineering, National University of Defense Technology, De-Ya Road 46, Kai-Fu District, \\ Hunan Province, Changsha 410073, China \\ Correspondence should be addressed to Jian Dong, dongjian@nudt.edu.cn
}

Received 22 August 2012; Accepted 15 October 2012

Academic Editor: Francisco Falcone

Copyright ( 92012 Jian Dong et al. This is an open access article distributed under the Creative Commons Attribution License, which permits unrestricted use, distribution, and reproduction in any medium, provided the original work is properly cited.

A novel scheme is developed for mitigating measurement biases in agile-beam polarimetric phased array weather radar. Based on the orthogonal Huygens source dual-polarized element model, a polarization measurement basis for planar polarimetric phased array radar (PPAR) is proposed. The proposed polarization basis is orthogonal to itself after a $90^{\circ}$ rotation along the array's broadside and can well measure the characteristics of dual-polarized element. With polarimetric measurements being undertaken in this polarization basis, the measurement biases caused by the unsymmetrical projections of dual-polarized element's fields onto the local horizontal and vertical directions of radiated beam can be mitigated. Polarimetric variables for precipitation estimation and classification are derived from the scattering covariance matrix in horizontal and vertical polarization basis. In addition, the estimates of these parameters based on the time series data acquired with the new polarization basis are also investigated. Finally, autocorrelation methods for both the alternate transmission and simultaneous reception mode and the simultaneous transmission and simultaneous reception mode are developed.

\section{Introduction}

The theory and application of polarimetric radar for weather sensing have been developed for decades [1,2]. Theoretically, any orthogonal polarization basis (e.g., horizontal and vertical linear, right hand and left hand circular, and $+45^{\circ}$ and $-45^{\circ}$ slant linear) can be used for fully polarimetric measurement. However, depending on the shape, size, and mean canting angle of hydrometeors, the linear $H / V$ (horizontal and vertical) polarization basis is the most common choice for current polarimetric weather radars with mechanically steered antenna, since it offers simpler, more direct, and accurate quantitative measurements of precipitations $[3,4]$. Polarimetric variables (e.g., the differential reflectivity $Z_{\mathrm{DR}}$, the total differential phase $\phi_{\mathrm{DP}}$, the copolar cross-correlation coefficient $\rho_{h v}$, and the linear depolarization ratio LDR) for precipitation estimation and classification have also been derived from the scattering covariance matrix in $H / V$ polarization basis [1-3]. The radar data acquired with other orthogonal polarization states need to be transformed to the $H / V$ polarization basis for the estimates of these parameters $[5,6]$.
Basically, there are two popular measurement modes for implementing the linear polarization basis. One is the simultaneous transmission and simultaneous reception (STSR) mode, while the other is the alternative transmission and simultaneous reception (ATSR) mode. Unless some orthogonal signal coding methods are used $[7,8]$, only copolar elements in scattering covariance matrix can be measured in the STSR mode. But there are indeed some benefits such as reduced statistical fluctuations, and no need to account for Doppler shifts and simplification of hardware, which made the STSR mode to be a common choice of recent polarimetric weather radar [3].

Polarimetric variables account for the differences of the $H$ and $V$ polarized fields which are reflected by or propagate through the hydrometeors. Since the shape of most hydrometeors is nearly spherical, the differences are usually very small. Accurate measurement of polarimetric variables is required to provide reliable information. There are many factors of biases in polarimetric measurement. One of the most critical factors comes from the nonideal polarization characteristics of an antenna, such as the mismatching of copular radiation and the limited isolation of cross-polar radiation. These 
effects were first examined in [9] for the ATSR mode. The requirements on the cross-polar isolation of antennas were investigated in [10], for both the ATSR and the STSR modes. More recent efforts reported in $[11,12]$ have considered the different cross-polar patterns of an antenna and given out compact forms of biases induced by the cross-polar radiation patterns and the unmatched copolar patterns.

In recent years, phased array radar technology has attracted wide attentions in weather radar community [1315]. Phased array antenna becomes an inevitable choice for next generation multifunction weather radar, due to its superiorities of faster scan speed, simultaneous multibeam, and adaptive beam forming. However, accurate polarimetric measurement with phased array antennas is still challenging [16]. The polarization characteristics of dual-polarized elements in phased array will vary in terms of beam-steering angle, which has been modeled theoretically by using orthogonal current sheets [17] and crossed infinitesimal dipoles $[16,18]$. The cross-polar radiation of phased array antenna has been derived theoretically in [18] and has been validated with measurements in [19]. As pointed out in [18], when beam is directed away from the broadside of planar phased array antenna, the misprojections of dual-polarized radiation fields onto the local $H$ and $V$ directions of the radiated beam would cause undesirable measurement biases, which are much larger than the intrinsic values of polarimetric variables. The solutions for correcting these biases have been discussed in [20], and two schemes by simultaneously adjusting the amplitude and phase of dual-polarized elements have been proposed. In order to achieve azimuth scan-invariant and high-accuracy weather measurements, a cylindrical configuration for polarimetric phased array radar has been proposed in [21]. Based on the interleaving sparse array (ISA) concept, an economical way of making projection corrections for planar phased array radar operated in alternate transmit-alternate receive (ATAR) mode has been proposed in [22]. The correction matrix approach proposed in $[16,18]$ has been generalized in [23-25] for the calibration of practical polarimetric phased array systems, where the imbalances and cross-couplings in transmitter and receiver $(\mathrm{T} / \mathrm{R})$ modules, the cross-coupling between antenna elements, and the polarization characteristics of practical antenna elements are included.

The existing schemes for mitigating measurement biases aimed at mimicking the polarimetric state of a mechanically steered beam in conventional $H / V$ polarization basis by simultaneously adjusting the feeding voltages of dualpolarized elements $[18,20]$. However, accurately adjusting transmitting power needs a highly linear power amplifier in the $T / R$ module, which is difficult to implement and will cause very low power efficiency. In contrast, a novel polarization basis for PPAR is proposed in this paper to fulfill polarimetric measurement. The proposed polarization basis has a superiority that is rotational symmetry along the array's broadside. The radiated fields of dual-polarized element can be projected symmetrically in the new polarization basis through the whole scan volume. Thereby, the synthesized polar beam pattern can be easily matched in all beamsteering directions, and the adjustments to the amplitudes

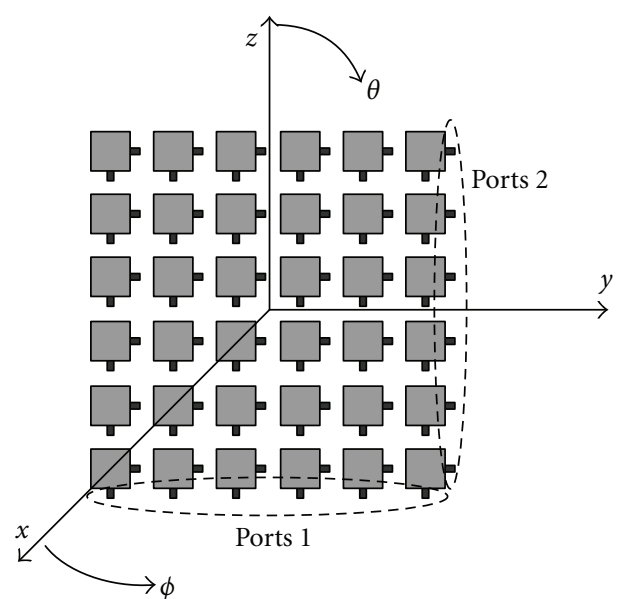

FIGURE 1: Illustration of $y, z$ plane located planner phased array antenna comprising dual-polarized radiation elements.

and phases of the feeding voltages to the dual-polarized elements can be avoided. However, as polarimetric variables for precipitation estimation and classification are derived from the scattering covariance matrix in conventional $H / V$ polarization basis, in order to retrieve these parameters based on the time series data acquired with the new polarization basis, autocorrelation methods for both the ATSR and the STSR modes are also investigated.

The rest of this paper is organized as follows. In Section 2, a new polarization measurement basis for planar phased array antenna is introduced based on the orthogonal Huygens source dual-polarized element model, and the benefits of this new basis for fulfilling polarimetric measurement are analyzed. The methods of retrieving scattering covariance matrix and polarimetric variables based on the time series data in the proposed polarization basis are investigated in Sections 3 and 4, respectively. Section 3 focuses on the ATSR mode, while Section 4 focuses on the STSR mode. Conclusions and perspectives are presented in Section 5.

\section{Theory Development}

As shown in Figure 1, a planar phased array antenna is located in the $y, z$ plane in the coordinate system. Each duallinear-polarized radiation element in the array has two ports named as port 1 and port 2, respectively. From the polarimetric measurement viewpoint, the purpose of this array design is to radiate orthogonal polarized fields throughout a scan volume, one from each port. However, in practice, the orthogonality of radiation fields excited by the two ports often cannot always be maintained through the whole scan volume. In order to measure this nonorthogonality and undertake polarimetric measurement, a predefined orthogonal polarization basis (i.e., two unit polarized vectors which are orthogonal to each other) needs to be selected in advance. Polarimetric radars often use the horizontal $(\phi$ direction for taking the $x, y$ plane as ground plane) and socalled "vertical" (negative $\theta$ direction which is only vertical at 0 elevation angle) polarization states for atmospheric 
observations. The $H / V$ polarization basis works well to radars with mechanically steered antennas, since the beam is always at the boresight, where the copolar patterns can be easily designed to match well and the cross-polar patterns are commonly at enough low level. However, in the case of planar dual-polarized phased array antennas, as most dual-linear-polarized elements have rotation symmetrical radiation characteristic with respect to the two feeding ports (i.e., the antenna radiates the same field when excited from port 1 as when excited from port 2 except that it is rotated $90^{\circ}$ about the broadside direction), the $H / V$ polarization basis cannot measure this symmetry since the $\theta$ or $\phi$ direction is not orthogonal to itself after a $90^{\circ}$ rotation along the array's broadside (i.e., normal to the array face). Consequently, when beams are steered away from the broadside, unsymmetrical projections of the electric fields excited by the two ports onto the local horizontal and vertical directions of radiated beam are induced. The measurement biases caused by these misprojections can be much larger than the intrinsic values of polarimetric variables [18]. On the other hand, if polarimetric measurements can be undertaken in a new polarization basis which is rotational symmetry about the array's broadside and can well measure the polarization characteristics of dual-polarized element, then the measurement biases can be mitigated. Thereby, the point is weather a new polarization basis can be defined from an "ideal" dual-polarized element, which radiates orthogonal fields in the whole scan volume when excited by two ports.

2.1. Orthogonal Huygens Source Element Model. Theoretically, a Huygens source can be modeled by a pair of crossed infinitesimal electrical and magnetic current sources (dipoles), which are taken to be at the same location and with the same radiation intensity [26, 27]. A dual-polarized element comprising two Huygens sources is located at origin with the PPAR array face in the $y, z$ plane, shown in Figure 2. One Huygens source is composed of a $y$-directed electrical current source $\left(\mathbf{I}_{e 1}=\hat{\mathbf{a}}_{y} I_{e l} l\right)$ and a $z$-directed magnetic current source $\left(\mathbf{I}_{m 1}=\hat{\mathbf{a}}_{z} I_{m 1} l\right)$, while another one is composed of a $z$-directed electrical current source $\left(\mathbf{I}_{e 2}=\widehat{\mathbf{a}}_{z} I_{e 2} 1\right)$ and a negative $y$-directed magnetic current source $\left(\mathbf{I}_{m 2}=\right.$ $\left.-\hat{\mathbf{a}}_{y} I_{m 2} l\right)$. The length of these current sources is infinitesimal, and $\lambda$ is the radar wavelength. They are considered to be orthogonal since these two Huygens sources have a relation of $90^{\circ}$ rotation around $x$-axis.

The radiation fields of electrical and magnetic current sources can be solved with the aid of auxiliary potential functions [28, Ch3]. The electric fields radiated by Huygens source comprising $\mathbf{I}_{e 1}$ and $\mathbf{I}_{m 1}$ and Huygens source comprising $\mathbf{I}_{e 2}$ and $\mathbf{I}_{m 2}$ are

$$
\begin{aligned}
& \mathbf{E}_{1}=-\frac{j k_{0} \eta M_{1}}{4 \pi r} e^{-j k_{0} r}\left[\hat{\mathbf{a}}_{\phi}(\sin \theta+\cos \phi)+\hat{\mathbf{a}}_{\theta} \cos \theta \sin \phi\right], \\
& \mathbf{E}_{2}=\frac{j k_{0} \eta M_{2}}{4 \pi r} e^{-j k_{0} r}\left[-\hat{\mathbf{a}}_{\phi} \cos \theta \sin \phi+\hat{\mathbf{a}}_{\theta}(\sin \theta+\cos \phi)\right],
\end{aligned}
$$

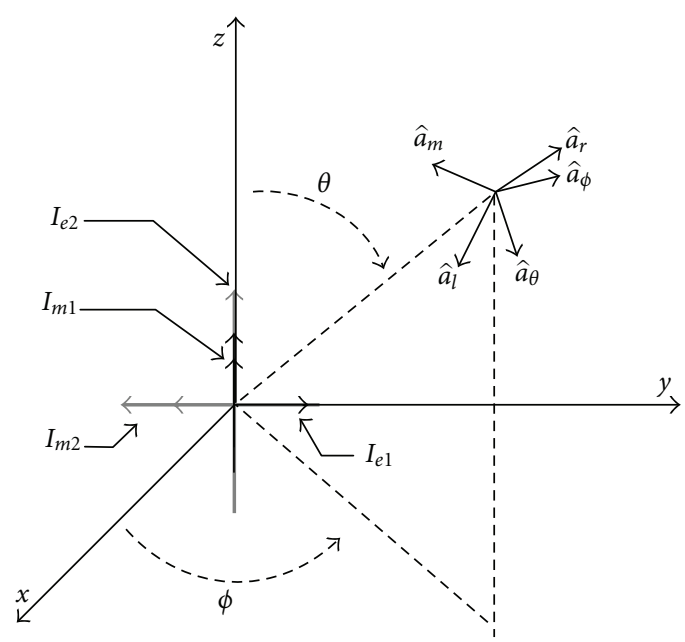

FIGURE 2: Spherical coordinate system for electric fields radiating form orthogonal Huygens sources.

where $k_{0}=2 \pi / \lambda$, and $\eta$ is the intrinsic impedance of the radiation medium and equals $\sqrt{\mu / \varepsilon}$. $\varepsilon$, and $\mu$ are the permittivity and permeability of the radiation medium. The radiated fields of electrical and magnetic current sources in one Huygens source should have the same magnitude and phase $[26,27]$. Thereby, $M_{1}=I_{e 1} l=I_{m 1} l / \eta$ and $M_{2}=I_{e 2} l_{2}=$ $I_{m 2} l_{2} / \eta$.

From (1) and (2), it can be verified that

$$
\mathbf{E}_{1} \cdot \mathbf{E}_{2}=0,
$$

where "." denotes the scalar product. Equation (3) is suitable for any values of $\theta, \phi$. It indicates that electric fields radiated by the orthogonal Huygens sources are orthogonal in any beam direction. Therefore, we can think that the orthogonal Huygens source is an "ideal" dual-polarized element for polarimetric measurement.

In order to classify different hydrometeors species, polarimetric weather radar often uses the horizontally $\left(\hat{\mathbf{a}}_{h}=\hat{\mathbf{a}}_{\phi}\right)$ and so-called "vertically" $\left(\hat{\mathbf{a}}_{v}=-\hat{\mathbf{a}}_{\theta}\right)$ polarized directions as orthogonal polarization measurement basis. Substituting $\hat{\mathbf{a}}_{h}$ for $\hat{\mathbf{a}}_{\phi}$ and $\hat{\mathbf{a}}_{v}$ for $-\hat{\mathbf{a}}_{\theta}$ in (1) and (2), respectively, the radiation fields of orthogonal Huygens source in $H / V$ polarization basis are represented as

$$
\begin{aligned}
& \mathbf{E}_{1}=E_{t 1}\left[-\widehat{\mathbf{a}}_{h}(\sin \theta+\cos \phi)+\hat{\mathbf{a}}_{v} \cos \theta \sin \phi\right], \\
& \mathbf{E}_{2}=E_{t 2}\left[-\hat{\mathbf{a}}_{h} \cos \theta \sin \phi-\widehat{\mathbf{a}}_{v}(\sin \theta+\cos \phi)\right],
\end{aligned}
$$

where

$$
E_{t q}=\frac{j k_{0} \eta M_{q}}{4 \pi r} e^{-j k_{0} r}
$$

is the electric field intensity transmitted along the direction of array broadside $\left(\theta=90^{\circ}, \phi=0^{\circ}\right)$. The subscript $q(1$ or 2$)$ denotes the $q$ th Huygens source. 
2.2. New Orthogonal Polarization Basis. As $\mathbf{E}_{1}$ and $\mathbf{E}_{2}$ are orthogonal at any far-field position, two new unit polarization vectors $\hat{\mathbf{a}}_{l}$ and $\hat{\mathbf{a}}_{m}$ can be defined as

$$
\begin{gathered}
\hat{\mathbf{a}}_{l}=\frac{1}{N_{\mathrm{or}}}\left[-\hat{\mathbf{a}}_{h}(\sin \theta+\cos \phi)+\hat{\mathbf{a}}_{v} \cos \theta \sin \phi\right], \\
\hat{\mathbf{a}}_{m}=\frac{1}{N_{\mathrm{or}}}\left[-\hat{\mathbf{a}}_{h} \cos \theta \sin \phi-\hat{\mathbf{a}}_{v}(\sin \theta+\cos \phi)\right],
\end{gathered}
$$

where

$$
N_{\text {or }}=N_{\text {or }}(\theta, \phi)=\sqrt{(\sin \theta+\cos \phi)^{2}+\cos ^{2} \theta \sin ^{2} \phi}
$$

is the normalized coefficient. $\hat{\mathbf{a}}_{l}$ and $\hat{\mathbf{a}}_{m}$ are also orthogonal and composing a new polarization basis. We denote this basis as $L / M$ polarization basis compared with the conventional $H / V$ polarization basis. The relation between the unit $L / M$ polarization and the unit $H / V$ polarization vectors can be expressed via a transform matrix

$$
\left[\begin{array}{c}
\hat{\mathbf{a}}_{l} \\
\hat{\mathbf{a}}_{m}
\end{array}\right]=\mathbf{U}_{y-z}\left[\begin{array}{l}
\hat{\mathbf{a}}_{h} \\
\hat{\mathbf{a}}_{v}
\end{array}\right],
$$

where

$$
\mathbf{U}_{y-z}=\frac{1}{N_{\mathrm{or}}}\left[\begin{array}{cc}
-(\sin \theta+\cos \phi) & \cos \theta \sin \phi \\
-\cos \theta \sin \phi & -(\sin \theta+\cos \phi)
\end{array}\right]
$$

is the polarization basis rotation matrix for planar array located in the $y, z$ plane. It has the property of

$$
\mathbf{U}_{y-z}^{T}=\mathbf{U}_{y-z}^{-1} .
$$

2.3. Backscattering Matrix. Substituting (6) into (1) and (7) into (2), respectively, electric fields radiated by two orthogonal Huygens sources in $L / M$ basis are

$$
\begin{gathered}
\mathbf{E}_{1}=E_{l}^{i} \widehat{\mathbf{a}}_{l}=E_{t 1} N_{\mathrm{or}} \hat{\mathbf{a}}_{l}, \\
\mathbf{E}_{2}=E_{m}^{i} \widehat{\mathbf{a}}_{m}=E_{t 2} N_{\mathrm{or}} \hat{\mathbf{a}}_{m},
\end{gathered}
$$

where $E_{l}^{i}$ and $E_{m}^{i}$ denote the $\hat{\mathbf{a}}_{l}$ and $\hat{\mathbf{a}}_{m}$ polarized incident field intensities at the hydrometeors. The relationship between the incident field intensities and the exciting currents can be expressed in matrix form as

$$
\left[\begin{array}{c}
E_{l}^{i} \\
E_{m}^{i}
\end{array}\right]=N_{\text {or }}\left[\begin{array}{c}
E_{t 1} \\
E_{t 2}
\end{array}\right]=N_{\text {or }} \frac{j k_{0} \eta}{4 \pi r} e^{-j k_{0} r}\left[\begin{array}{c}
M_{1} \\
M_{2}
\end{array}\right] .
$$

$N_{\text {or }}$ in (13) is the normalized copolar field radiation pattern of Huygens source in $L / M$ polarization basis, and the crosspolar pattern of "ideal" Huygens source in $L / M$ polarization basis is always zero.

The backscattering field $\left(\mathbf{E}^{s}\right)$ by hydrometeors can be expressed as

$$
\mathbf{E}^{s}=\left[\begin{array}{c}
E_{l}^{s} \\
E_{m}^{s}
\end{array}\right]=\mathbf{S}_{l m}^{(b)}\left[\begin{array}{c}
E_{l}^{i} \\
E_{m}^{i}
\end{array}\right]
$$

where $\mathbf{S}_{l m}^{(b)}$ is the intrinsic backscatter matrix of hydrometeors in $L / M$ polarization basis

$$
\mathbf{S}_{l m}^{(b)}=\left[\begin{array}{cc}
s_{l l}^{(b)} & s_{l m}^{(b)} \\
s_{m l}^{(b)} & s_{m m}^{(b)}
\end{array}\right] .
$$

Since the scattering medium is reciprocal, the off-diagonal terms in (15) are equal. The transform between $\mathbf{S}_{l m}^{(b)}$ and the intrinsic backscatter matrix in $H / V$ polarization basis $\left(\mathbf{S}_{h v}^{(b)}\right)$ can be expressed as

$$
\begin{aligned}
\mathbf{S}_{l m}^{(b)} & =\mathbf{U}_{y-z} \mathbf{S}_{h v}^{(b)} \mathbf{U}_{y-z}^{-1}=\mathbf{U}_{y-z} \mathbf{S}_{h v}^{(b)} \mathbf{U}_{y-z}^{T} \\
& =\mathbf{U}_{y-z}\left[\begin{array}{cc}
s_{h h}^{(b)} & s_{h v}^{(b)} \\
s_{v h}^{(b)} & s_{v v}^{(b)}
\end{array}\right] \mathbf{U}_{y-z}^{T} .
\end{aligned}
$$

The scattered field $E_{l}^{s}$ can be completely received by one Huygens source as they share the same polarization state, while the scattered field $E_{m}^{s}$ can also be completely received by the other one. So electric fields received by two Huygens sources can be expressed as

$$
\begin{aligned}
{\left[\begin{array}{c}
E_{l}^{r} \\
E_{m}^{r}
\end{array}\right] } & =\frac{e^{-j k_{0} r}}{r}\left[\begin{array}{c}
E_{l}^{s} \\
E_{m}^{s}
\end{array}\right]=\frac{e^{-j k_{0} r}}{r} \mathbf{S}_{l m}^{(b)}\left[\begin{array}{c}
E_{l}^{i} \\
E_{m}^{i}
\end{array}\right] \\
& =N_{\text {or }} \frac{e^{-j k_{0} r}}{r} \mathbf{S}_{l m}^{(b)}\left[\begin{array}{c}
E_{t 1} \\
E_{t 2}
\end{array}\right] .
\end{aligned}
$$

2.4. Propagation and Cross-Polarization Effects. The discussion so far deliberately ignored propagation effects. In practice, when electromagnetic waves propagate through precipitation, there could be differential attenuations and differential phase shifts between the two differential polarized waves. Both attenuations and phases shifts along propagation path affect the received fields. Therefore, corrections for these factors are necessary [1-4]. Accounting to the propagation effects, (17) should be generalized as

$$
\left[\begin{array}{c}
E_{l}^{r} \\
E_{m}^{r}
\end{array}\right]=N_{\text {or }} \frac{e^{-j k_{0} r}}{r} \mathbf{T}_{l m}^{T} \mathbf{S}_{l m}^{(b)} \mathbf{T}_{l m}\left[\begin{array}{c}
E_{t 1} \\
E_{t 2}
\end{array}\right],
$$

where

$$
\mathbf{T}_{l m}=\left[\begin{array}{cc}
T_{l l} & T_{l m} \\
T_{m l} & T_{m m}
\end{array}\right],
$$

is the transmission matrix in $L / M$ polarization basis. The relation between $\mathbf{T}_{l m}$ and the transmission matrix in $H / V$ polarization basis $\left(\mathbf{T}_{h v}\right)$ is

$$
\mathbf{T}_{l m}=\mathbf{U}_{y-z} \mathbf{T}_{h \nu} \mathbf{U}_{y-z}^{-1}=\mathbf{U}_{y-z} \mathbf{T}_{h \nu} \mathbf{U}_{y-z}^{T} .
$$

Since most hydrometeors have an axis of symmetry near vertical, $H$ or $V$ linearly polarized waves practically remain in the same pure polarization state as they propagate through precipitation, and there is no depolarization of $H$ and $V$ waves [3]. The transmission matrix accounting for the extra phase shift and attenuation induced by the hydrometeors is a diagonal matrix

$$
\mathbf{T}_{h v}=\left[\begin{array}{cc}
T_{h h} & 0 \\
0 & T_{v v}
\end{array}\right]
$$


It indicates that the $H$ and $V$ components of the wave propagate independently. For weather radar operating at long wavelengths (e.g., $10 \mathrm{~cm}$ ), attenuation is rather small and is usually negligible [3]. Consequently, (21) is simplified as

$$
\mathbf{T}_{h v}=\left[\begin{array}{cc}
T_{h h} & 0 \\
0 & T_{v v}
\end{array}\right]=\left[\begin{array}{cc}
e^{-j k_{h} r} & 0 \\
0 & e^{-j k_{v} r}
\end{array}\right]
$$

where $k_{h}$ and $k_{v}$ are the "perturbation" component of the free space propagation constant (i.e., $k_{0}$ ) due to the presence of the medium. One of the most important polarimetric variables for rainfall estimation is the specific differential phase [1], which is defined as $K_{\mathrm{DP}}=k_{h}-k_{v}$. The two-way differential propagation phase is defined as $\phi_{\mathrm{dp}}=2 K_{\mathrm{DP}} r$, which denotes the two-way propagation phase difference of $H$ and $V$ polarized waves along a distance of $r$.

Substituting (16) and (20) into (18) and using the equations $\mathbf{U}_{y-z}^{T}=\mathbf{U}_{y-z}^{-1}$ and $\mathbf{T}_{h v}=\mathbf{T}_{h v}^{T}$, the electric fields received by two Huygens sources are

$$
\begin{aligned}
{\left[\begin{array}{c}
E_{l}^{r} \\
E_{m}^{r}
\end{array}\right]=} & N_{\mathrm{or}} \frac{e^{-j k_{0} r}}{r} \mathbf{U}_{y-z} \mathbf{T}_{h v}^{T} \mathbf{U}_{y-z}^{T} \mathbf{U}_{y-z} \mathbf{S}_{h v}^{(b)} \\
& \times \mathbf{U}_{y-z}^{-1} \mathbf{U}_{y-z} \mathbf{T}_{h v} \mathbf{U}_{y-z}^{-1}\left[\begin{array}{c}
E_{t 1} \\
E_{t 2}
\end{array}\right] \\
= & N_{\mathrm{or}} \frac{e^{-j k_{0} r}}{r} \mathbf{U}_{y-z} \mathbf{T}_{h v} \mathbf{S}_{h v}^{(b)} \mathbf{T}_{h v} \mathbf{U}_{y-z}^{T}\left[\begin{array}{c}
E_{t 1} \\
E_{t 2}
\end{array}\right] \\
= & N_{\mathrm{or}} \frac{e^{-j k_{0} r}}{r} \mathbf{U}_{y-z} \mathbf{S}_{h v}^{\prime} \mathbf{U}_{y-z}^{T}\left[\begin{array}{c}
E_{t 1} \\
E_{t 2}
\end{array}\right],
\end{aligned}
$$

where $\mathbf{S}_{h v}^{\prime}$ denotes the combined backscattering and transmission matrix in $H / V$ polarization basis [18]. It links the forward- and back-propagating dual-polarized electric fields in $H / V$ polarization basis at the location of the antenna, and it is given by

$$
\begin{aligned}
\mathbf{S}_{h v}^{\prime} & =\mathbf{T}_{h v} \mathbf{S}_{h v}^{(b)} \mathbf{T}_{h v} \\
& =\left[\begin{array}{cc}
T_{h h}^{2} s_{h h}^{(b)} & T_{h h} T_{v v} s_{h v}^{(b)} \\
T_{h h} T_{v v} s_{v h}^{(b)} & T_{v v}^{2} s_{v v}^{(b)}
\end{array}\right] \\
& =\left[\begin{array}{ll}
s_{h h}^{\prime} & s_{h v}^{\prime} \\
s_{v h}^{\prime} & s_{v v}^{\prime}
\end{array}\right] .
\end{aligned}
$$

The off-diagonal terms of $\mathbf{S}_{h v}^{\prime}$ are equal $\left(s_{h v}^{\prime}=s_{v h}^{\prime}\right)$. Equation (23) is very important, since it shows theoretically that it is not necessary to remove the propagation effects before polarization basis transformation [6]. So the transform from $\mathbf{S}_{h v}^{\prime}$ to the combined backscattering and transmission matrix in $L / M$ polarization basis $\left(\mathbf{S}_{l m}^{\prime}\right)$ is

$$
\mathbf{S}_{l m}^{\prime}=\left[\begin{array}{cc}
s_{l l}^{\prime} & s_{l m}^{\prime} \\
s_{m l}^{\prime} & s_{m m}^{\prime}
\end{array}\right]=\mathbf{T}_{l m}^{T} \mathbf{S}_{l m}^{(b)} \mathbf{T}_{l m}=\mathbf{U}_{y-z} \mathbf{S}_{h v}^{\prime} \mathbf{U}_{y-z}^{T}
$$

2.5. Receiving Voltage Equation. Electric fields received by an antenna are transformed by receivers into baseband voltages.
If the relative gains and the phase difference of dual polarization receivers are calibrated, baseband voltages received and transformed by two Huygens sources can be represented as

$$
\begin{aligned}
{\left[\begin{array}{c}
V_{l}^{r} \\
V_{m}^{r}
\end{array}\right] } & =N_{\mathrm{or}}^{2} \mathbf{S}_{l m}^{\prime}\left[\begin{array}{l}
E_{t 1} \\
E_{t 2}
\end{array}\right] \\
& =N_{\mathrm{or}}^{2} \mathbf{U}_{y-z} \mathbf{S}_{h v}^{\prime} \mathbf{U}_{y-z}^{T}\left[\begin{array}{c}
E_{t 1} \\
E_{t 2}
\end{array}\right],
\end{aligned}
$$

where $V_{l}^{r}$ and $V_{m}^{r}$ are the received baseband voltages of $L$ and $M$ polarized signals, respectively. $N_{\text {or }}^{2}$ in (26) is the normalized copolar power radiation pattern of Huygens source antenna in $L / M$ polarization basis. It is used to account the antenna's gain decreasing when the beam's direction is away from the array's broadside.

Furthermore, (26) indicates that $\mathbf{S}_{h v}^{\prime}$ can be fully recovered without any bias if $\mathbf{S}_{l m}^{\prime}$ can be accurately measured. The transform from $\mathbf{S}_{l m}^{\prime}$ to $\mathbf{S}_{h v}^{\prime}$ is straightforward

$$
\mathbf{S}_{h v}^{\prime}=\mathbf{U}_{y-z}^{T} \mathbf{S}_{l m}^{\prime} \mathbf{U}_{y-z} .
$$

However, the returned signal from a radar resolution volume filled with hydrometeors often fluctuates with time, which can be described in terms of the Doppler velocity spectrum of the scatterers $[1,2]$. Equation (27) is valid only if one can carry out simultaneous measurements of the four time series, $s_{l l}^{\prime}, s_{m l}^{\prime}, s_{m m}^{\prime}$ and $s_{l m}^{\prime}$. When radar system operates at the ATSR mode, the pair $s_{l l}^{\prime}, s_{m l}^{\prime}$, and pair $s_{m m}^{\prime}, s_{l m}^{\prime}$ are measured at alternative pulse repetition intervals, and the coupling between the Doppler and the polarimetric effect prevents directly using (27) to retrieve $\mathbf{S}_{h v}^{\prime}$. This coupling issue will be discussed and resolved in Section 3. In contrast, the Doppler and polarimetric effects are not coupled in the STSR mode. This mode is based on the approximation that the intrinsic scattering matrix is diagonal, which is valid for most meteorological conditions in $H$ and $V$ linear polarization observations. However, when STSR mode measurements are undertaken in the proposed $L / M$ linear polarization basis, the off-diagonal terms of $\mathbf{S}_{l m}^{\prime}$ would have nonzero value in most observing directions even if $\mathbf{S}_{h v}^{\prime}$ is diagonal, and the four terms in $\mathbf{S}_{l m}^{\prime}$ cannot be measured separately. Only with the assumption that $\mathbf{S}_{h v}^{\prime}$ is diagonal, diagonal terms of $\mathbf{S}_{h v}^{\prime}$ can be recovered by postprocessing the $L$ and $M$ polarization received signals. This issue will be discussed in Section 4 .

2.6. Practical Antenna Element Consideration. Although orthogonal Huygens source is a theoretically dual-polarized element model, the design and realization of electrically small antennas which have similar radiation characteristics as Huygens source have been studied recently [27, 29]. If this kind of antenna is used as radiation element of planar PPAR, polarimetric measurements can be undertaken in the proposed $L / M$ polarization basis without obvious measurement biases.

For some popular antenna elements such as dualpolarized microstrip antennas [30], the proposed orthogonal polarization basis is also suitable. As aforementioned, 
when polarimetric planar phased array composed of dualpolarized elements is designed to radiate orthogonal polarizations throughout a scan volume, the radiation characteristics of antenna element are often symmetrical with respect to the two feeding ports. In order to measure the orthogonality of the polarizations from the two ports, the logical choice of orthogonal polarization basis for describing copolar and cross-polar radiation patterns is the basis which is orthogonal to itself after a $90^{\circ}$ rotation about array broadside. The proposed $L / M$ polarization basis belongs to this case, while the $H / V$ polarization basis does not. The matching of copolar radiation characteristics (i.e., the copolar radiation patterns should have the same main-beam shape, and the orthogonal copolar radiation fields excited by each port with equal power should have the same intensity) is very important for highly accurate polarimetric measurement. However, when $H / V$ polarization basis is used for planar phased array, in order to keep the transmit $H$ and $V$ fields to be the same as fields from mechanically steering beam, the voltage (power) fed to each port needs to be adjusted as a function of beam directions $[20,24,25]$. If antenna element of planar phased array has rotation symmetrical radiation characteristic, the copolar radiation pattern in the proposed $L / M$ polarization basis can match well, which would make the calibration process more straightforward.

With the electric and magnetic currents composing orthogonal Huygens sources placed along the $x$-, $y$-axes, the analogous polarization basis for planer array located in the $x$, $y$ plane can be represented as

$$
\begin{aligned}
{\left[\begin{array}{c}
\hat{\mathbf{a}}_{\mathrm{co}} \\
\hat{\mathbf{a}}_{\text {cross }}
\end{array}\right] } & =\left[\begin{array}{cc}
\cos \phi & \sin \phi \\
-\sin \phi & \cos \phi
\end{array}\right]\left[\begin{array}{l}
\hat{\mathbf{a}}_{\phi} \\
\hat{\mathbf{a}}_{\theta}
\end{array}\right] \\
& =\mathbf{U}_{x-y}\left[\begin{array}{c}
\hat{\mathbf{a}}_{\phi} \\
\hat{\mathbf{a}}_{\theta}
\end{array}\right],
\end{aligned}
$$

where $\mathbf{U}_{x-y}$ is the polarization basis rotation matrix for planar array located in the $x, y$ plane. The $\hat{\mathbf{a}}_{\text {co }}$ and $\hat{\mathbf{a}}_{\text {cross }}$ unit vectors are exactly the Ludwig's III definition of linear reference and cross-polarization, which have already been proposed in the Ludwig's classical paper for describing polarization purity of antenna patterns [31]. Since this definition was formulated mathematically in 1973, its usage had become commonplace in antenna measurement community to measure and describe the copolar and crosspolar patterns of linear polarized antennas [32]. When using this kind of predefined polarization basis as the orthogonal transmitting/receiving polarization states of planar PPAR, the radiation characteristics of dual-polarized element can be well described, and the measurement biases caused by the misprojection of the copolar and cross-polar fields onto the local $H$ and $V$ directions of the radiated beam [18] can be mitigated. If mutual couplings between elements in a phased array are modeled with active (embedded) element patterns [33], the relative cross-polar level of synthesized beam is just the relative cross-polar level of active element patterns in the same beam direction, which is the primary reason for the polarization measurement errors by the antenna.

As described in [20], in order to produce a desired $H$ or $V$ polarized beam independent of direction, the transmitting fields of each polarization by dual-polarized element need to be adjusted simultaneously. Since each copolar radiation fields in the new polarization basis can be considered to be only corresponding with one of the element's feeding ports, all the matter of nonideal polarization can be reduced to the cross-polarized radiation fields. If the cross-polar pattern's maximal level in the whole scan volume is low enough to produce negligible measurement biases, each polarization can be synthesized individually, which is another benefit of the proposed polarization basis. The cross-polarized fields of conventional dual-polarized element (e.g., dual-polarized microstrip antenna) in the new polarization basis can reach its maximum in the diagonal far-field planes. If these fields are strong enough and need to be taken into account in polarization beam synthesis, then the ISA technique [22] can be used here to cancel the cross-polarization of the main beam since each polarization is individually controlled.

The proposed polarization basis provides a new choice of accurate polarimetric measurement for planer phased array. The estimating methods for these polarimetric variables by the new polarization measurement will be discussed in next two sections.

\section{Polarimetric Variables Measurement at ATSR Mode}

The methods of retrieving scattering covariance matrix and polarimetric variables based on the new polarization measurements in ATSR mode are presented in this section. The definition of polarimetric variables for meteorological research and some approximation of backscatter and propagation characteristics of hydrometeors are reviewed at first. Then two ways of polarimetric variables measurement are discussed.

\subsection{Scattering Covariance Matrix and Polarimetric Variables.} Since meteorological targets are composed of an ensemble of reshuffling hydrometeors that are located randomly in space, the total return from a radar resolution volume fluctuates with time [6]. The mean scattering matrix is no longer sufficient to completely characterize this scattering medium. Thereby, the scattering covariance matrix is necessary. The definition of the scattering covariance matrix $[1,2,34]$ is

$$
\left[\begin{array}{ccc}
\left\langle\left|s_{h h}^{(b)}\right|^{2}\right\rangle & \left\langle s_{h v}^{(b)} s_{h h}^{(b)^{*}}\right\rangle & \left\langle s_{v v}^{(b)} s_{h h}^{(b)}\right\rangle \\
\left\langle s_{h h}^{(b)} s_{h v}^{(b)^{*}}\right\rangle & \left\langle\left|s_{h v}^{(b)}\right|^{2}\right\rangle & \left\langle s_{v v}^{(b)} s_{h v}^{(b)}\right\rangle \\
\left\langle s_{h h}^{(b)} s_{v v}^{(b)}\right\rangle & \left\langle s_{h v}^{(b)} s_{v v}^{(b)}\right\rangle & \left\langle\left|s_{v v}^{(b)}\right|^{2}\right\rangle
\end{array}\right],
$$

where $s_{x y}^{(b)}(x, y=h, v)$ is the term of intrinsic backscatter matrix in $H / V$ polarization basis, and the angle brackets denote ensemble averaging.

Most terms of the covariance matrix have been used by themselves or in combination with others to infer properties of the scattering hydrometeors. The popular polarimetric variables that are derived from the covariance are $Z_{\mathrm{DR}}$, 
$\rho_{h v}$, and LDR. The definitions of these polarimetric variables are

$$
\begin{gathered}
Z_{\mathrm{DR}}=10 \log \left(\frac{\left\langle\left|s_{h h}^{(b)}\right|^{2}\right\rangle}{\left\langle\left|s_{v v}^{(b)}\right|^{2}\right\rangle}\right), \\
\rho_{h v}(0)=\frac{\left\langle s_{v v}^{(b)} s_{h h}^{(b)}{ }^{*}\right\rangle}{\sqrt{\left\langle\left|s_{h h}^{(b)}\right|^{2}\right\rangle\left\langle\left|s_{v v}^{(b)}\right|^{2}\right\rangle}}, \\
\mathrm{LDR}_{h}=10 \log \left(\frac{\left\langle\left|s_{v h}^{(b)}\right|^{2}\right\rangle}{\left\langle\left|s_{h h}^{(b)}\right|^{2}\right\rangle}\right), \\
\mathrm{LDR}_{v}=10 \log \left(\frac{\left\langle\left|s_{h v}^{(b)}\right|^{2}\right\rangle}{\left\langle\left|s_{v v}^{(b)}\right|^{2}\right\rangle}\right) .
\end{gathered}
$$

As the Rayleigh-Gans scattering theory is valid for hydrometeors at long wavelength (e.g., $10 \mathrm{~cm}$ ), the differences of backscattering phase angles by most hydrometeors are usually very small [35]. These phase differences are negligible, and the off-diagonal terms of (29) are considered to be real numbers. When propagation effects are taken into account, $s_{x y}^{\prime}$ needs to be substituted for $s_{x y}^{(b)}(x, y=h, v)$ in (30). Attenuation along propagation path in most precipitation media is rather small and can also be neglected at long wavelength [3], Thereby, the values of $Z_{\mathrm{DR}}$ and LDR with propagation effects will not be changed. Due to the differential propagation phase shift of $H$ and $V$ polarization waves, the copolar cross-correlation coefficient with propagation effects can be represented as

$$
\rho_{h v}^{\prime}(0)=\frac{\left\langle s_{v v}^{\prime} s_{h h}^{\prime}{ }^{*}\right\rangle}{\sqrt{\left\langle\left|s_{h h}^{\prime}\right|^{2}\right\rangle\left\langle\left|s_{v v}^{\prime}\right|^{2}\right\rangle}}=e^{j \phi_{\mathrm{DP}}} \rho_{h v}(0),
$$

where $\phi_{\text {DP }}$ is the two-way differential propagation phase which has been introduced before.

Due to the relative motion and random wobbling of hydrometeors in the radar resolution volume, there are Doppler phase shift and spectral broadening in the correlation of different time sampled echoes $[1,2]$. Since the relative motions and positions of scatterers are independent of their sizes and shapes at low elevation angles, various correlation coefficients between components of either polarization at one sample time and components at another sample time can be expressed as a product of coefficients due to Doppler spread and due to polarization effects [36]. For radar with alternating transmit polarization, $s_{v v}^{\prime}$ and $s_{h h}^{\prime}$ are measured at alternate pulse repetition intervals. The copolar crosscorrelation coefficient can be expressed as

$$
\begin{aligned}
\rho_{h v}^{\prime}\left(T_{s}\right) & =\frac{\left\langle s_{v v}^{\prime}(2 i+1) s_{h h}^{\prime}{ }^{*}(2 i)\right\rangle}{\sqrt{\left\langle\left|s_{h h}^{\prime}(2 i)\right|^{2}\right\rangle\left\langle\left|s_{v v}^{\prime}(2 i+1)\right|^{2}\right\rangle}} \\
& \approx e^{j \omega_{d} T_{s}}\left|\rho\left(T_{s}\right)\right| \frac{\left\langle s_{v v}^{\prime} s_{h h}^{\prime} *\right.}{\sqrt{\left\langle\left|s_{h h}^{\prime}\right|^{2}\right\rangle\left\langle\left|s_{v v}^{\prime}\right|^{2}\right\rangle}} \\
& =e^{j\left(\omega_{d} T_{s}+\phi_{\mathrm{DP}}\right)}\left|\rho\left(T_{s}\right)\right| \rho_{h v}(0),
\end{aligned}
$$

where $T_{s}$ is the pulse repetition time, $i$ is an integer implicitly multiplying $T_{s}$, and $\rho\left(T_{s}\right)$ is the correlation coefficient at lag $T_{s}$ due to the Doppler spread. $\omega_{d} T_{s}$ is the mean Doppler phase shift, which corresponds the mean velocity $(\hat{v})$ of hydrometeors in the radar resolution volume with $\omega_{d} T_{s}=$ $-2 T_{s} k_{0} \hat{v}$.

Since most weather echoes have Gaussian shape correlation coefficients (Doppler spectrum), the magnitude of Doppler spectrum satisfy

$$
\left|\rho\left(T_{s}\right)\right|=\left(\left|\rho\left(2 T_{s}\right)\right|\right)^{0.25}
$$

where $\rho\left(2 T_{s}\right)$ is the correlation coefficient at lag $2 T_{s}$ due to the Doppler spread. Also, there may be some differences in Doppler phase/spectrum of various polarization correlations at the same lag [35], which will be ignored in this paper.

3.2. Polarimetric Variables Measurement by Estimates of Instantaneous Scattering Matrices. Consider the ATSR mode and two consecutive times defined with indices $2 i$ and $2 i+1$, where $i$ is an integer implicitly multiplying the pulse repetition time $T_{s}$. At time $2 i$, a pure $L$ polarized field is transmitted followed by a pure $M$ polarized field at time $2 i+1$. From (26), the received baseband voltage alternates as follows.

At time (2i)

$$
\begin{aligned}
{\left[\begin{array}{c}
V_{l}^{r}(2 i) \\
V_{m}^{r}(2 i)
\end{array}\right] } & =N_{\mathrm{or}}^{2} \mathbf{S}_{l m}^{\prime}(2 i)\left[\begin{array}{c}
E_{t 1} \\
0
\end{array}\right] \\
& =N_{\mathrm{or}}^{2} \mathbf{U}_{y-z} \mathbf{S}_{h v}^{\prime}(2 i) \mathbf{U}_{y-z}^{T}\left[\begin{array}{c}
E_{t 1} \\
0
\end{array}\right]
\end{aligned}
$$

At time $(2 i+1)$

$$
\begin{aligned}
{\left[\begin{array}{c}
V_{l}^{r}(2 i+1) \\
V_{m}^{r}(2 i+1)
\end{array}\right] } & =N_{\mathrm{or}}^{2} \mathbf{S}_{l m}^{\prime}(2 i+1)\left[\begin{array}{c}
0 \\
E_{t 2}
\end{array}\right] \\
& =N_{\mathrm{or}}^{2} \mathbf{U}_{y-z} \mathbf{S}_{h v}^{\prime}(2 i+1) \mathbf{U}_{y-z}^{T}\left[\begin{array}{c}
0 \\
E_{t 2}
\end{array}\right] .
\end{aligned}
$$

Substituting $\mathbf{S}_{l m}^{\prime}, \mathbf{S}_{h v}^{\prime}$, and $\mathbf{U}_{y-z}$ into (34) and (35), these voltages can be expressed as follows. 
At time (2i)

$$
\begin{aligned}
V_{l}^{r}(2 i)= & N_{\mathrm{or}}^{2} s_{l l}^{\prime}(2 i) E_{t 1} \\
= & N_{\mathrm{or}}^{2}\left[a^{2} s_{h h}^{\prime}(2 i)+b^{2} s_{v v}^{\prime}(2 i)-2 a b s_{h v}^{\prime}(2 i)\right] E_{t 1}, \\
V_{m}^{r}(2 i)= & N_{\mathrm{or}}^{2} s_{m l}^{\prime}(2 i) E_{t 1} \\
= & N_{\mathrm{or}}^{2}\left[a b s_{h h}^{\prime}(2 i)-a b s_{v v}^{\prime}(2 i)+\left(a^{2}-b^{2}\right) s_{h v}^{\prime}(2 i)\right] \\
& \times E_{t 1} .
\end{aligned}
$$

At time $(2 i+1)$

$$
\begin{gathered}
V_{l}^{r}(2 i+1)=N_{\mathrm{or}}^{2} s_{l m}^{\prime}(2 i+1) E_{t 2} \\
=N_{\mathrm{or}}^{2}\left[a b s_{h h}^{\prime}(2 i+1)-a b s_{v v}^{\prime}(2 i+1)\right. \\
\left.\quad+\left(a^{2}-b^{2}\right) s_{h v}^{\prime}(2 i+1)\right] E_{t 2}, \\
V_{m}^{r}(2 i+1)=N_{\mathrm{or}}^{2} s_{m m}^{\prime}(2 i+1) E_{t 2} \\
=N_{\mathrm{or}}^{2}\left[b^{2} s_{h h}^{\prime}(2 i+1)+a^{2} s_{v v}^{\prime}(2 i+1)\right. \\
\left.\quad+2 a b s_{h v}^{\prime}(2 i+1)\right] E_{t 2},
\end{gathered}
$$

where the constants $a$ and $b$ are $a=(\sin \theta+\cos \phi) / N_{\text {or }}$, $b=(\cos \theta \sin \phi) / N_{\text {or }} . a$ and $b$ are the diagonal and offdiagonal terms of $\mathbf{U}_{y-z}$ and have a relation of $a^{2}+b^{2}=1$. The time series $s_{l l}^{\prime}(2 i), s_{m l}^{\prime}(2 i), s_{l m}^{\prime}(2 i+1)$, and $s_{m m}^{\prime}(2 i+1)$ can be directly calculated from (36), (37), (38), and (39), respectively. Since these time series are not measured at the same time, they cannot make up of the instantaneous combined backscattering and transmission matrix in $L / M$ polarization basis. Therefore, (27) cannot be used directly.

The question here is whether an instantaneous scattering matrix can be estimated from such alternate time series. This problem has been discussed in [6], and we represent here for completeness. If the pulse repetition time $T_{s}$ is within half of the correlation threshold time as defined in [6], the instantaneous combined backscattering and transmission matrix $\widetilde{\mathbf{S}}_{l m}^{\prime}$ can be constructed accurately and has a form as

$$
\tilde{\mathbf{S}}_{l m}^{\prime}=\left[\begin{array}{cc}
\tilde{s}_{l l}^{\prime}(2 i+1) & s_{l m}^{\prime}(2 i+1) \\
\tilde{s}_{m l}^{\prime}(2 i+1) & s_{m m}^{\prime}(2 i+1)
\end{array}\right],
$$

where $\tilde{s}_{l l}^{\prime}(2 i+1)$ and $\tilde{s}_{m l}^{\prime}(2 i+1)$ are interpolated copolar and cross-polar terms at time $2 i+1$. By invoking reciprocity for the case of backscatter, the cross-polar term $\tilde{s}_{m l}^{\prime}(2 i+1)$ can be estimated immediately as

$$
\tilde{s}_{m l}^{\prime}(2 i+1)=s_{l m}^{\prime}(2 i+1) \text {. }
$$

The estimation problem is to determine the amplitude and phase of $\tilde{s}_{l l}^{\prime}(2 i+1)$. The amplitude of $\tilde{s}_{l l}^{\prime}(2 i+1)$ can be estimated by a simple interpolation as

$$
\left|\tilde{s}_{l l}^{\prime}(2 i+1)\right|=\frac{\left|s_{l l}^{\prime}(2 i)\right|+\left|s_{l l}^{\prime}(2 i+2)\right|}{2} .
$$

The phase of $\widetilde{s}_{l l}^{\prime}(2 i+1)$ can be estimated as

$$
\arg \left[\tilde{s}_{l l}^{\prime}(2 i+1)\right]=\arg \left[s_{m m}^{\prime}(2 i+1)\right]+\psi_{C}^{L}(2 i+1)
$$

where $\psi_{C}^{L}(2 i+1)$ is the total copolar differential phase of $L$ and $M$ polarization states at time $2 i+1$, and it can be estimated as

$$
\begin{aligned}
& \psi_{C}^{L}(2 i+1) \\
& \quad=\frac{1}{2} \arg \left\{\left[s_{l l}^{\prime}(2 i+2) s_{m m}^{\prime} *(2 i+1)\right]\left[s_{m m}^{\prime}(2 i+1) s_{l l}^{\prime *}(2 i)\right]^{*}\right\} .
\end{aligned}
$$

Equation (44) is just the $i$ th estimate according to the well known estimator for total mean copolar differential phase $\left(\psi_{C}^{L}\right)$, as given in [6],

$$
\psi_{C}^{L}=\arg \left(R_{C 1}^{L}{ }^{*} R_{C 2}^{L}\right)
$$

where

$$
\begin{gathered}
R_{C 1}^{L}=\frac{1}{N} \sum_{i=0}^{N-1} s_{l l}^{\prime *}(2 i) s_{m m}^{\prime}(2 i+1), \\
R_{C 2}^{L}=\frac{1}{N-1} \sum_{i=0}^{N-2} s_{l l}^{\prime}(2 i+2) s_{m m}^{\prime} *(2 i+1),
\end{gathered}
$$

are the two estimates of cross-copolar covariance at lag 1.

Assuming that the relative phase difference between dual polarized transmitting and receiving channel of the radar are calibrated (or accounted), the instantaneous combined backscattering and transmission matrix can then be constructed as

$$
\begin{aligned}
& \tilde{\mathbf{S}}_{l m}^{\prime}(2 i+1) \\
& \quad=\left[\begin{array}{cc}
\left|\tilde{s}_{l l}^{\prime}(2 i+1)\right| e^{j \psi_{C}^{L}(2 i+1)} e^{\arg \left[s_{m m}^{\prime}(2 i+1)\right]} & s_{l m}^{\prime}(2 i+1) \\
s_{l m}^{\prime}(2 i+1) & s_{m m}^{\prime}(2 i+1)
\end{array}\right] .
\end{aligned}
$$

This matrix can be transformed by (27) to the $H / V$ polarization basis. Polarimetric variables for precipitation estimation can then be calculated.

3.3. Polarimetric Variables Measurement by Estimates of Powers and Correlations. In this subsection, we will demonstrate how polarimetric variables can be calculated by operating on estimates of powers and correlations of the $L$ and $M$ polarization echoes. Assuming that an alternate sequence of 
$2 N+1$ pulses is transmitted and received, according to (36) (39), the power estimates from the even sequence (subscript e) $2 i$ are

$$
\begin{aligned}
P_{l e} & =\frac{1}{N} \sum_{i=0}^{N-1}\left|V_{l}^{r}(2 i)\right|^{2} \\
& =N_{\mathrm{or}}^{4}\left|E_{t 1}\right|^{2} \frac{1}{N} \sum_{i=0}^{N-1}\left|s_{l l}^{\prime}(2 i)\right|^{2} \\
& =N_{\mathrm{or}}^{4}\left|E_{t 1}\right|^{2}\left\langle\left|s_{l l}^{\prime}\right|^{2}\right\rangle, \\
P_{m e} & =\frac{1}{N} \sum_{i=0}^{N-1}\left|V_{m}^{r}(2 i)\right|^{2} \\
& =N_{\mathrm{or}}^{4}\left|E_{t 1}\right|^{2} \frac{1}{N} \sum_{i=0}^{N-1}\left|s_{m l}^{\prime}(2 i)\right|^{2} \\
& =N_{\mathrm{or}}^{4}\left|E_{t 1}\right|^{2}\left\langle\left|s_{m l}^{\prime}\right|^{2}\right\rangle,
\end{aligned}
$$

and the correlation estimate is

$$
\begin{aligned}
R_{l, m e} & =\frac{1}{N} \sum_{i=0}^{N-1} V_{l}^{r *}(2 i) V_{m}^{r}(2 i) \\
& =N_{\mathrm{or}}^{4}\left|E_{t 1}\right|^{2} \frac{1}{N} \sum_{i=0}^{N-1} s_{l l}^{\prime *}(2 i) s_{m l}^{\prime}(2 i) \\
& =N_{\mathrm{or}}^{4}\left|E_{t 1}\right|^{2}\left\langle s_{l l}^{\prime *} s_{m l}^{\prime}\right\rangle .
\end{aligned}
$$

The power estimates form the odd sequence (subscript $o$ ) $2 i+$ 1 are

$$
\begin{aligned}
P_{l o} & =\frac{1}{N} \sum_{i=0}^{N-1}\left|V_{l}^{r}(2 i+1)\right|^{2} \\
& =N_{\mathrm{or}}^{4}\left|E_{t 2}\right|^{2} \frac{1}{N} \sum_{i=0}^{N-1}\left|s_{l m}^{\prime}(2 i+1)\right|^{2} \\
& =N_{\mathrm{or}}^{4}\left|E_{t 2}\right|^{2}\left\langle\left|s_{l m}^{\prime}\right|^{2}\right\rangle, \\
P_{m o} & =\frac{1}{N} \sum_{i=0}^{N-1}\left|V_{m}^{r}(2 i+1)\right|^{2} \\
& =N_{\mathrm{or}}^{4}\left|E_{t 2}\right|^{2} \frac{1}{N} \sum_{i=0}^{N-1}\left|s_{m m}^{\prime}(2 i+1)\right|^{2} \\
& =N_{\mathrm{or}}^{4}\left|E_{t 2}\right|^{2}\left\langle\left|s_{m m}^{\prime}\right|^{2}\right\rangle,
\end{aligned}
$$

and the correlation estimate is

$$
\begin{aligned}
R_{l, m o} & =\frac{1}{N} \sum_{i=0}^{N-1} V_{l}^{r *}(2 i+1) V_{m}^{r}(2 i+1) \\
& =N_{\mathrm{or}}^{4}\left|E_{t 2}\right|^{2} \frac{1}{N} \sum_{i=0}^{N-1} s_{l m}^{\prime}{ }^{*}(2 i+1) s_{m m}^{\prime}(2 i+1) \\
& =N_{\mathrm{or}}^{4}\left|E_{t 2}\right|^{2}\left\langle s_{l m}^{\prime}{ }^{*} s_{m m}^{\prime}\right\rangle .
\end{aligned}
$$

The two cross-correlations of the even and odd sequences are

$$
\begin{aligned}
R_{l e, m o} & =\frac{1}{N} \sum_{i=0}^{N-1} V_{l}^{r *}(2 i) V_{m}^{r}(2 i+1) \\
& =N_{\mathrm{or}}^{4} E_{t 1}^{*} E_{t 2} \frac{1}{N} \sum_{i=0}^{N-1} s_{l l}^{\prime *}(2 i) s_{m m}^{\prime}(2 i+1), \\
R_{m o, l e} & =\frac{1}{N} \sum_{i=0}^{N-1} V_{m}^{r} *(2 i+1) V_{l}^{r}(2 i+2) \\
& =N_{\mathrm{or}}^{4} E_{t 1} E_{t 2}^{*} \frac{1}{N} \sum_{i=0}^{N-1} s_{m m}^{\prime} *(2 i+1) s_{l l}^{\prime}(2 i+2) .
\end{aligned}
$$

The subscripts le, mo in (53) denote that the correlation of $L$ polarization received even voltage sequence with $M$ polarization receiving odd voltage sequence, where the sequence corresponding to the subscript before comma is always conjugated and having leading time in the correlation. With the same notation, other cross-correlations (i.e., $R_{l e, l o}, R_{l o, l e}$, $R_{m e, m o}, R_{m o, m e}, R_{m e, l o}$, and $\left.R_{l o, m e}\right)$ can be similarly defined. As indicated before, $N_{\text {or }}^{2}$ is the normalized copolar power radiation pattern of Huygens source antenna, and $E_{t q}(q=$ $1,2)$ is the electric field transmitted along array broadside. For practical antenna elements, the measured (or computed) copolar active power radiation pattern in $L / M$ polarization basis should be substituted for $N_{\text {or }}^{2}$ in (48) and (54). Assuming that the relative gains and the phase differences of two receivers are calibrated and removed from baseband voltages, the magnitude and phase imbalances of $E_{t 1}$ and $E_{t 1}$ need to be measured by some calibration processing. Then the following power and correlations can be derived:

$$
\begin{aligned}
p_{l e} & =\frac{1}{N} \sum_{i=0}^{N-1}\left|\frac{V_{l}^{r}(2 i)}{N_{\mathrm{or}}^{2} E_{t 1}}\right|^{2}=\frac{P_{l e}}{N_{\mathrm{or}}^{4}\left|E_{t 1}\right|^{2}} \\
& =\frac{1}{N} \sum_{i=0}^{N-1}\left|s_{l l}^{\prime}(2 i)\right|^{2}=\left\langle\left|s_{l l}^{\prime}\right|^{2}\right\rangle,
\end{aligned}
$$




$$
\begin{aligned}
& p_{m e}=\frac{1}{N} \sum_{i=0}^{N-1}\left|\frac{V_{m}^{r}(2 i)}{N_{\mathrm{or}}^{2} E_{t 1}}\right|^{2}=\frac{P_{m e}}{N_{\mathrm{or}}^{4}\left|E_{t 1}\right|^{2}} \\
& =\frac{1}{N} \sum_{i=0}^{N-1}\left|s_{m l}^{\prime}(2 i)\right|^{2}=\left\langle\left|s_{m l}^{\prime}\right|^{2}\right\rangle \text {, } \\
& p_{l o}=\frac{1}{N} \sum_{i=0}^{N-1}\left|\frac{V_{l}^{r}(2 i+1)}{N_{\mathrm{or}}^{2} E_{t 2}}\right|^{2}=\frac{P_{l o}}{N_{\mathrm{or}}^{4}\left|E_{t 2}\right|^{2}} \\
& =\frac{1}{N} \sum_{i=0}^{N-1}\left|s_{l m}^{\prime}(2 i+1)\right|^{2}=\left\langle\left|s_{l m}^{\prime}\right|^{2}\right\rangle \text {, } \\
& p_{m o}=\frac{1}{N} \sum_{i=0}^{N-1}\left|\frac{V_{m}^{r}(2 i+1)}{N_{\mathrm{or}}^{2} E_{t 2}}\right|^{2}=\frac{P_{m o}}{N_{\mathrm{or}}^{4}\left|E_{t 2}\right|^{2}} \\
& =\frac{1}{N} \sum_{i=0}^{N-1}\left|s_{m m}^{\prime}(2 i+1)\right|^{2}=\left\langle\left|s_{m m}^{\prime}\right|^{2}\right\rangle \text {, } \\
& r_{l, m e}=\frac{1}{N} \sum_{i=0}^{N-1}\left(\frac{V_{l}^{r}(2 i)}{N_{\mathrm{or}}^{2} E_{t 1}}\right)^{*}\left(\frac{V_{m}^{r}(2 i)}{N_{\mathrm{or}}^{2} E_{t 1}}\right) \\
& =\frac{R_{l, m e}}{N_{\mathrm{or}}^{4}\left|E_{t 1}\right|^{2}}=\frac{1}{N} \sum_{i=0}^{N-1} s_{l l}^{\prime *}(2 i) s_{m l}^{\prime}(2 i) \\
& =\left\langle s_{l l}^{\prime *} s_{m l}^{\prime}\right\rangle \text {, } \\
& r_{l, m o}=\frac{1}{N} \sum_{i=0}^{N-1}\left(\frac{V_{l}^{r}(2 i+1)}{N_{\mathrm{or}}^{2} E_{t 2}}\right)^{*}\left(\frac{V_{m}^{r}(2 i+1)}{N_{\mathrm{or}}^{2} E_{t 2}}\right) \\
& =\frac{R_{l, m o}}{N_{\mathrm{or}}^{4}\left|E_{t 2}\right|^{2}}=\frac{1}{N} \sum_{i=0}^{N-1} s_{l m}^{\prime} *(2 i+1) s_{m m}^{\prime}(2 i+1) \\
& =\left\langle s_{l m}^{\prime} * s_{m m}^{\prime}\right\rangle, \\
& r_{l e, m o}=\frac{1}{N} \sum_{i=0}^{N-1}\left(\frac{V_{l}^{r}(2 i)}{N_{\mathrm{or}}^{2} E_{t 1}}\right)^{*}\left(\frac{V_{m}^{r}(2 i+1)}{N_{\mathrm{or}}^{2} E_{t 2}}\right) \\
& =\frac{R_{l e, m o}}{N_{\mathrm{or}}^{4} E_{t 1}^{*} E_{t 2}}=\frac{1}{N} \sum_{i=0}^{N-1} s_{l l}^{\prime} *(2 i) s_{m m}^{\prime}(2 i+1) \\
& \approx\left\langle s_{l l}^{\prime}{ }^{*} s_{m m}^{\prime}\right\rangle\left|\rho\left(T_{s}\right)\right| e^{j \omega_{d} T_{s}}, \\
& r_{m e, l o}=\frac{1}{N} \sum_{i=0}^{N-1}\left(\frac{V_{m}^{r}(2 i)}{N_{\mathrm{or}}^{2} E_{t 1}}\right)^{*}\left(\frac{V_{l}^{r}(2 i+1)}{N_{\mathrm{or}}^{2} E_{t 2}}\right) \\
& =\frac{R_{m e, l o}}{N_{\mathrm{or}}^{4} E_{t 1}^{*} E_{t 2}}=\frac{1}{N} \sum_{i=0}^{N-1} s_{m l}^{\prime} *(2 i) s_{l m}^{\prime}(2 i+1) \\
& \approx\left\langle s_{m l}^{\prime}{ }^{*} s_{l m}^{\prime}\right\rangle\left|\rho\left(T_{s}\right)\right| e^{j \omega_{d} T_{s}},
\end{aligned}
$$

where (61), (62) have included the approximation that the Doppler effects and the polarization effects can be separated at low elevation angles. Other cross-correlations (i.e., $r_{l e, l o}$, $r_{m e, m o}, r_{m o, l e}, r_{l o, l e}, r_{l o, m e}$, and $\left.r_{m o, m e}\right)$ can also be calculated.
With reciprocity condition $\left(s_{m l}^{\prime}=s_{l m}^{\prime}\right)$, the following equations can be derived:

$$
\begin{gathered}
p_{m e}=\left\langle\left|s_{m l}^{\prime}\right|^{2}\right\rangle=\left\langle\left|s_{l m}^{\prime}\right|^{2}\right\rangle=p_{l o}, \\
r_{m e, l o}=\left\langle s_{m l}^{\prime}{ }^{*} s_{l m}^{\prime}\right\rangle\left|\rho\left(T_{s}\right)\right| e^{j \omega_{d} T_{s}} \\
=\left\langle\left|s_{m l}^{\prime}\right|^{2}\right\rangle\left|\rho\left(T_{s}\right)\right| e^{j \omega_{d} T_{s}} \\
=p_{m e}\left|\rho\left(T_{s}\right)\right| e^{j \omega_{d} T_{s}} .
\end{gathered}
$$

If there is enough power in the cross-polar receiving channel, (64) can be used to estimate the Doppler spread and the mean Doppler velocity. The magnitude of correlation coefficient can be estimated as

$$
\left|\rho\left(T_{s}\right)\right|=\frac{\left|r_{m e, l o}\right|}{p_{m e}} .
$$

The mean Doppler phase shift can be estimated as

$$
\omega_{d} T_{s}=\arg \left(r_{m e, l o}\right)
$$

and the ambiguity of $\omega_{d} T_{s}$ corresponds to the sampling at time $T_{s}$.

Another method for estimating $\left|\rho\left(T_{s}\right)\right|$ and $\omega_{d} T_{s}$ is using the correlation of either the odd or even copolar sequence at lag $2 T_{s}$. For the copolar even sequence, we have

$$
\begin{aligned}
r_{l e}\left(2 T_{s}\right) & =\frac{1}{N-1} \sum_{i=0}^{N-2}\left(\frac{V_{l}^{r}(2 i)}{N_{\mathrm{or}}^{2} E_{t 1}}\right)^{*}\left(\frac{V_{l}^{r}(2 i+2)}{N_{\mathrm{or}}^{2} E_{t 1}}\right) \\
& =\frac{1}{N-1} \sum_{i=0}^{N-2} s_{l l}^{\prime *}(2 i) s_{l l}^{\prime}(2 i+2) \\
& =p_{l e}\left|\rho\left(2 T_{s}\right)\right| e^{j 2 \omega T_{s}} .
\end{aligned}
$$

For the Gaussian-shaped Doppler spectrum, $\left|\rho\left(T_{s}\right)\right|$ can be estimated as

$$
\left|\rho\left(T_{s}\right)\right|=\left(\left|\rho\left(2 T_{s}\right)\right|\right)^{0.25}=\left[\frac{\left|r_{l e}\left(2 T_{s}\right)\right|}{p_{l e}}\right]^{0.25}
$$

and $\omega_{d} T_{s}$ can be estimated as

$$
\omega_{d} T_{s}=\frac{1}{2} \arg \left[r_{l e}\left(2 T_{s}\right)\right]
$$

with an ambiguity corresponding to the sampling at $2 T_{s}$.

Furthermore, we will demonstrate how polarimetric variables defined in (30) can be estimated from the powers and correlations of (55)-(62). Two types of precipitation media are considered. One is a medium that has off-diagonal backscattering terms, while the other does not.

3.3.1. Nondiagonal Backscattering Matrix. The transform from $S_{l m}^{\prime}$ to $S_{h v}^{\prime}$ has been given in (27), we rewrite it here with expansions 


$$
\begin{aligned}
{\left[\begin{array}{ll}
s_{h h}^{\prime} & s_{h v}^{\prime} \\
s_{v h}^{\prime} & s_{v v}^{\prime}
\end{array}\right] } & =\left[\mathbf{U}_{y-z}\right]^{T}\left[\mathbf{S}_{l m}^{\prime}\right]\left[\mathbf{U}_{y-z}\right] \\
& =\left[\begin{array}{cc}
a^{2} s_{l l}^{\prime}+a b s_{m l}^{\prime}+a b s_{l m}^{\prime}+b^{2} s_{m m}^{\prime} & -a b s_{l l}^{\prime}-b^{2} s_{m l}^{\prime}+a^{2} s_{l m}^{\prime}+a b s_{m m}^{\prime} \\
-a b s_{l l}^{\prime}+a^{2} s_{m l}^{\prime}-b^{2} s_{l m}^{\prime}+a b s_{m m}^{\prime} & b^{2} s_{l l}^{\prime}-a b s_{m l}^{\prime}-a b s_{l m}^{\prime}+a^{2} s_{m m}^{\prime}
\end{array}\right] .
\end{aligned}
$$

With the approximation that Doppler effects and polarization effects are separated, the four covariance elements $\left\langle\left|s_{h h}^{\prime}\right|^{2}\right\rangle,\left\langle\left|s_{v v}^{\prime}\right|^{2}\right\rangle,\left\langle\left|s_{v h}^{\prime}\right|^{2}\right\rangle$, and $\left\langle s_{h h}^{\prime}{ }^{*} s_{v v}^{\prime}\right\rangle$ can be calculated from power and correlations defined in (55)-(62) as

$$
\begin{aligned}
& \left\langle\left|s_{h h}^{\prime}\right|^{2}\right\rangle=\left\langle\left|a^{2} s_{l l}^{\prime}+a b s_{m l}^{\prime}+a b s_{l m}^{\prime}+b^{2} s_{m m}^{\prime}\right|^{2}\right\rangle \\
& =a^{4}\left\langle\left|s_{l l}^{\prime}\right|^{2}\right\rangle+4 a^{2} b^{2}\left\langle\left|s_{m l}^{\prime}\right|^{2}\right\rangle+b^{4}\left\langle\left|s_{m m}^{\prime}\right|^{2}\right\rangle \\
& +4 a^{3} b \operatorname{Re}\left(\left\langle s_{l l}^{\prime *} s_{m l}^{\prime}\right\rangle\right)+2 a^{2} b^{2} \operatorname{Re}\left(\left\langle s_{l l}^{\prime *} s_{m m}^{\prime}\right\rangle\right) \\
& +4 a b^{3} \operatorname{Re}\left(\left\langle s_{l m}^{\prime}{ }^{*} s_{m m}^{\prime}\right\rangle\right) \\
& =a^{4} p_{l e}+4 a^{2} b^{2} p_{m e}+b^{4} p_{m o}+4 a^{3} b \operatorname{Re}\left(r_{l, m e}\right) \\
& +4 a b^{3} \operatorname{Re}\left(r_{l, m o}\right)+\frac{2 a^{2} b^{2} \operatorname{Re}\left(r_{l e, m o}\right)}{\left|\rho\left(T_{s}\right)\right| \cos \left(\omega_{d} T_{s}\right)}, \\
& \left\langle\left|s_{v v}^{\prime}\right|^{2}\right\rangle=\left\langle\left|b^{2} s_{l l}^{\prime}-a b s_{m l}^{\prime}-a b s_{l m}^{\prime}+a^{2} s_{m m}^{\prime}\right|^{2}\right\rangle \\
& =b^{4}\left\langle\left|s_{l l}^{\prime}\right|^{2}\right\rangle+4 a^{2} b^{2}\left\langle\left|s_{m l}^{\prime}\right|^{2}\right\rangle+a^{4}\left\langle\left|s_{m m}^{\prime}\right|^{2}\right\rangle \\
& -4 a b^{3} \operatorname{Re}\left(\left\langle s_{l l}^{\prime *} s_{m l}^{\prime}\right\rangle\right)+2 a^{2} b^{2} \operatorname{Re}\left(\left\langle s_{l l}^{\prime}{ }^{*} s_{m m}^{\prime}\right\rangle\right) \\
& -4 a^{3} b \operatorname{Re}\left(\left\langle s_{l m}^{\prime}{ }^{*} s_{m m}^{\prime}\right\rangle\right) \\
& =b^{4} p_{l e}+4 a^{2} b^{2} p_{m e}+a^{4} p_{m o}-4 a b^{3} \operatorname{Re}\left(r_{l, m e}\right) \\
& -4 a^{3} b \operatorname{Re}\left(r_{l, m o}\right)+\frac{2 a^{2} b^{2} \operatorname{Re}\left(r_{l e, m o}\right)}{\left|\rho\left(T_{s}\right)\right| \cos \left(\omega_{d} T_{s}\right)},
\end{aligned}
$$$$
\left\langle\left|s_{v h}^{\prime}\right|^{2}\right\rangle=\left\langle\left|-a b s_{l l}^{\prime}+a^{2} s_{m l}^{\prime}-b^{2} s_{l m}^{\prime}+a b s_{m m}^{\prime}\right|^{2}\right\rangle
$$$$
=a^{2} b^{2}\left\langle\left|s_{l l}^{\prime}\right|^{2}\right\rangle+\left(a^{2}-b^{2}\right)^{2}\left\langle\left|s_{m l}^{\prime}\right|^{2}\right\rangle
$$$$
+a^{2} b^{2}\left\langle\left|s_{m m}^{\prime}\right|^{2}\right\rangle-2 a b\left(a^{2}-b^{2}\right)
$$$$
\times \operatorname{Re}\left(\left\langle s_{l l}^{\prime *} s_{m l}^{\prime}\right\rangle\right)-2 a^{2} b^{2} \operatorname{Re}\left(\left\langle s_{l l}^{\prime *} s_{m m}^{\prime}\right\rangle\right)
$$$$
+2 a b\left(a^{2}-b^{2}\right) \operatorname{Re}\left(\left\langle s_{l m}^{\prime}{ }^{*} s_{m m}^{\prime}\right\rangle\right)
$$

$$
\begin{aligned}
= & a^{2} b^{2} p_{l e}+\left(a^{2}-b^{2}\right)^{2} p_{m e}+a^{2} b^{2} p_{m o} \\
- & 2 a b\left(a^{2}-b^{2}\right) \operatorname{Re}\left(r_{l, m e}\right)+2 a b\left(a^{2}-b^{2}\right) \\
\times & \operatorname{Re}\left(r_{l, m o}\right)-\frac{2 a^{2} b^{2} \operatorname{Re}\left(r_{l e, m o}\right)}{\left|\rho\left(T_{s}\right)\right| \cos \left(\omega_{d} T_{s}\right)}, \\
\left\langle s_{h h}^{\prime}{ }^{*} s_{v v}^{\prime}\right\rangle & \\
= & \left\langle\left[a^{2} s_{l l}^{\prime}+a b s_{m l}^{\prime}+a b s_{l m}^{\prime}+b^{2} s_{m m}^{\prime}\right]^{*}\right. \\
& \left.\times\left[b^{2} s_{l l}^{\prime}-a b s_{m l}^{\prime}-a b s_{l m}^{\prime}+a^{2} s_{m m}^{\prime}\right]\right\rangle \\
= & a^{2} b^{2}\left\langle\left|s_{l l}^{\prime}\right|^{2}\right\rangle-4 a^{2} b^{2}\left\langle\left|s_{m l}^{\prime}\right|^{2}\right\rangle+a^{2} b^{2}\left\langle\left|s_{m m}^{\prime}\right|^{2}\right\rangle \\
& -2 a^{3} b\left\langle s_{l l}^{\prime} s_{m l}^{\prime}\right\rangle+2 a b^{3}\left\langle s_{l l}^{\prime *} s_{m l}^{\prime}\right\rangle^{*} \\
& +2 a^{3} b\left\langle s_{l m}^{\prime}{ }^{*} s_{m m}^{\prime}\right\rangle-2 a b^{3}\left\langle s_{l m}^{\prime}{ }^{*} s_{m m}^{\prime}\right\rangle \\
& +a^{4}\left\langle s_{l l}^{\prime *} s_{m m}^{\prime}\right\rangle+b^{4}\left\langle s_{l l}^{\prime *} s_{m m}^{\prime}\right\rangle^{*} \\
= & a^{2} b^{2} p_{l e}-4 a^{2} b^{2} p_{m e}+a^{2} b^{2} p_{m o} \\
& -2 a^{3} b r_{l, m e}+2 a b^{3} r_{l, m e}^{*}+2 a^{3} b r_{l, m o} \\
& 2 a b^{3} r_{l, m o}^{*}+a^{4} r_{l e, m o}+b^{4} r_{l e, m o}^{*}, \\
& \\
& \\
&
\end{aligned}
$$

where the reciprocity of scattering matrix $\left(s_{m l}^{\prime}=s_{l m}^{\prime}\right)$ is used several times in these equations. The estimates of correlation coefficient $\left(\left|\rho\left(T_{s}\right)\right|\right)$ and mean Doppler phase shift $\left(\omega_{d} T\right)$ have been given before. When the attenuation along propagation path is ignored, $Z_{\mathrm{DR}}, \mathrm{LDR}$, and $\rho_{h v}^{\prime}$ can be computed as

$$
\begin{gathered}
Z_{\mathrm{DR}}=10 \log \left(\frac{\left\langle\left|s_{h h}^{(b)}\right|^{2}\right\rangle}{\left\langle\left|s_{v v}^{(b)}\right|^{2}\right\rangle}\right)=10 \log \left(\frac{\left\langle\left|s_{h h}^{\prime}\right|^{2}\right\rangle}{\left\langle\left|s_{v v}^{\prime}\right|^{2}\right\rangle}\right) \\
\mathrm{LDR}_{h}=10 \log \left(\frac{\left\langle\left|s_{v h}^{(b)}\right|^{2}\right\rangle}{\left\langle\left|s_{h h}^{(b)}\right|^{2}\right\rangle}\right)=10 \log \left(\frac{\left\langle\left|s_{v h}^{\prime}\right|^{2}\right\rangle}{\left\langle\left|s_{h h}^{\prime}\right|^{2}\right\rangle}\right) \\
\rho_{h v}^{\prime}=e^{j \phi_{\mathrm{DP}}} \rho_{h v}(0)=\frac{\left\langle s_{v v}^{\prime} s_{h h}^{\prime}{ }^{*}\right\rangle}{\sqrt{\left\langle\left|s_{h h}^{\prime}\right|^{2}\right\rangle\left\langle\left|s_{v v}^{\prime}\right|^{2}\right\rangle}} .
\end{gathered}
$$


Equation (74) can be used to estimate $\phi_{\mathrm{DP}}$ and $\rho_{h v}(0)$. With the approximation that all elements in backscattering matrix have the same phase angles, $\phi_{\text {DP }}$ can be estimated as

$$
\phi_{\mathrm{DP}}=\arg \left[\left\langle s_{v v}^{\prime} s_{h h}^{\prime *}\right\rangle\right]
$$

and $\rho_{h v}(0)$ can be estimated as

$$
\rho_{h v}(0)=\frac{\left|\left\langle s_{v v}^{\prime} s_{h h}^{\prime *}\right\rangle\right|}{\sqrt{\left\langle\left|s_{h h}^{\prime}\right|^{2}\right\rangle\left\langle\left|s_{v v}^{\prime}\right|^{2}\right\rangle}} .
$$

3.3.2. Diagonal Backscattering Matrix. When observing at low elevation angles, the off-diagonal terms of intrinsic backscattering matrix is very small (can be ignored) for most meteorological conditions in $H$ and $V$ linear polarization observations. Although the methods presented in last subsection are also suitable for this case, some simple but more effective methods for the estimates of polarimetric variables are presented here.

Since the off-diagonal terms of $\mathbf{S}_{h v}^{\prime}$ are considered to be zero $\left(s_{h v}^{\prime}=s_{v h}^{\prime}=0\right)$, the received baseband voltages are at time $(2 i)$

$$
\begin{aligned}
V_{l}^{r}(2 i) & =N_{\mathrm{or}}^{2} s_{l l}^{\prime}(2 i) E_{t 1} \\
& =N_{\mathrm{or}}^{2}\left[a^{2} s_{h h}^{\prime}(2 i)+b^{2} s_{v v}^{\prime}(2 i)\right] E_{t 1}, \\
V_{m}^{r}(2 i) & =N_{\mathrm{or}}^{2} s_{m l}^{\prime}(2 i) E_{t 1} \\
& =N_{\mathrm{or}}^{2}\left[a b s_{h h}^{\prime}(2 i)-a b s_{v v}^{\prime}(2 i)\right] E_{t 1},
\end{aligned}
$$

and at time $(2 i+1)$

$$
\begin{aligned}
V_{l}^{r}(2 i+1) & =N_{\mathrm{or}}^{2} s_{l m}^{\prime}(2 i+1) E_{t 2} \\
& =N_{\mathrm{or}}^{2}\left[a b s_{h h}^{\prime}(2 i+1)-a b s_{v v}^{\prime}(2 i+1)\right] E_{t 2}, \\
V_{m}^{r}(2 i+1) & =N_{\mathrm{or}}^{2} s_{m m}^{\prime}(2 i+1) E_{t 2} \\
& =N_{\mathrm{or}}^{2}\left[b^{2} s_{h h}^{\prime}(2 i+1)+a^{2} s_{v v}^{\prime}(2 i+1)\right] E_{t 2} .
\end{aligned}
$$

We can solve the $s_{h h}^{\prime}(2 i)$ and $s_{v v}^{\prime}(2 i)$ from (77) and (78)

$$
\begin{aligned}
& s_{h h}^{\prime}(2 i)=\frac{a V_{l}^{r}(2 i)+b V_{m}^{r}(2 i)}{a N_{\mathrm{or}}^{2} E_{t 1}}=\frac{\left[a s_{l l}^{\prime}(2 i)+b s_{m l}^{\prime}(2 i)\right]}{a}, \\
& s_{v v}^{\prime}(2 i)=\frac{b V_{l}^{r}(2 i)-a V_{m}^{r}(2 i)}{b N_{\mathrm{or}}^{2} E_{t 1}}=\frac{\left[b s_{l l}^{\prime}(2 i)-a s_{m l}^{\prime}(2 i)\right]}{b},
\end{aligned}
$$

and $s_{h h}^{\prime}(2 i+1)$ and $s_{v v}^{\prime}(2 i+1)$ from (79) and (80)

$$
\begin{aligned}
s_{h h}^{\prime}(2 i+1) & =\frac{a V_{l}^{r}(2 i+1)+b V_{m}^{r}(2 i+1)}{b N_{\mathrm{or}}^{2} E_{t 2}} \\
& =\frac{\left[a s_{l m}^{\prime}(2 i+1)+b s_{m m}^{\prime}(2 i+1)\right]}{b}, \\
s_{v v}^{\prime}(2 i+1) & =-\frac{b V_{l}^{r}(2 i+1)-a V_{m}^{r}(2 i+1)}{a N_{\mathrm{or}}^{2} E_{t 2}} \\
& =-\frac{\left[b s_{l m}^{\prime}(2 i+1)-a s_{m m}^{\prime}(2 i+1)\right]}{a} .
\end{aligned}
$$

Precipitation observation is often taken at low elevation angles, where the value of $\theta$ is approach $90^{\circ}$. Thereby, the coefficient $b=\cos \theta \sin \phi / N_{\text {or }}$ in (81) and (84) is very small. As $b$ is in denominator of (82) and (83), only (81) and (84) can be used here to avoid computational errors.

From the power estimates of (81) and (84), the covariance $\left\langle\left|s_{h h}^{\prime}\right|^{2}\right\rangle$ and $\left\langle\left|s_{v v}^{\prime}\right|^{2}\right\rangle$ can be derived as

$$
\begin{aligned}
\left\langle\left|s_{h h}^{\prime}\right|^{2}\right\rangle & =\frac{1}{N} \sum_{i=0}^{N-1}\left|s_{h h}^{\prime}(2 i)\right|^{2} \\
& =\frac{1}{a^{2}}\left[a^{2} p_{l e}+b^{2} p_{m e}+2 a b \operatorname{Re}\left(r_{l, m e}\right)\right], \\
\left\langle\left|s_{v v}^{\prime}\right|^{2}\right\rangle & =\frac{1}{N} \sum_{i=0}^{N-1}\left|s_{v v}^{\prime}(2 i+1)\right|^{2} \\
& =\frac{1}{a^{2}}\left[b^{2} p_{l o}+a^{2} p_{m o}-2 a b \operatorname{Re}\left(r_{l, m o}\right)\right] .
\end{aligned}
$$

Then $Z_{\mathrm{DR}}$ can be estimated by substituting (85) and (86) into (72).

The two cross-correlations of (81) and (84) can be calculated as

$$
\begin{aligned}
R_{a}\left(T_{s}\right)= & \frac{1}{N} \sum_{i=0}^{N-1} s_{h h}^{\prime}{ }^{*}(2 i) s_{v v}^{\prime}(2 i+1) \\
= & \frac{1}{a^{2}} \frac{1}{N} \sum_{i=0}^{N-1}\left[a s_{l l}^{\prime}(2 i)+b s_{m l}^{\prime}(2 i)\right]^{*} \\
& \times\left[-b s_{l m}^{\prime}(2 i+1)+a s_{m m}^{\prime}(2 i+1)\right] \\
= & \frac{1}{a^{2}}\left[-a b r_{l e, l o}+a b r_{m e, m o}+a^{2} r_{l e, m o}-b^{2} r_{m e, l o}\right], \\
R_{b}\left(T_{s}\right)= & \frac{1}{N} \sum_{i=0}^{N-1} s_{v v}^{\prime} *(2 i+1) s_{h h}^{\prime}(2 i+2) \\
= & \frac{1}{a^{2}} \frac{1}{N} \sum_{i=0}^{N-1}\left[-b s_{l m}^{\prime}(2 i+1)+a s_{m m}^{\prime}(2 i+1)\right] \\
& \times\left[a s_{l l}^{\prime}(2 i+2)+b s_{m l}^{\prime}(2 i+2)\right] \\
= & \frac{1}{a^{2}}\left[-a b r_{l o, l e}+a b r_{m o, m e}-b^{2} r_{l o, m e}+a^{2} r_{m o, l e}\right] .
\end{aligned}
$$


As indicated in [37], the argument of $R_{a}$ (87) equals the sum of the differential phase $\phi_{\mathrm{DP}}$ and the Doppler shift $\omega_{d} T_{s}$, whereas the argument of $R_{b}(88)$ is $-\phi_{\mathrm{DP}}+\omega_{d} T_{s}$. So the $\phi_{\mathrm{DP}}$ can be estimated using $\arg \left(R_{a} R_{b}^{*}\right) / 2$. The magnitudes of $R_{a}$ and $R_{b}$ can be represented as

$$
\begin{aligned}
& \left|R_{a}\left(T_{s}\right)\right|=\left|\left\langle s_{h h}^{\prime}{ }^{*} s_{v v}^{\prime}\right\rangle\right|\left|\rho\left(T_{s}\right)\right|, \\
& \left|R_{b}\left(T_{s}\right)\right|=\left|\left\langle s_{v v}^{\prime}{ }^{*} s_{h h}^{\prime}\right\rangle\right|\left|\rho\left(T_{s}\right)\right| .
\end{aligned}
$$

Although (89) and (90) are equal in mean, both of them would be estimated and averaged to reduce errors in practice. So $\left|\left\langle s_{h h}^{\prime}{ }^{*} s_{v v}^{\prime}\right\rangle\right|$ can be estimated as

$$
\left|\left\langle s_{h h}^{\prime}{ }^{*} s_{v v}^{\prime}\right\rangle\right|=\frac{\left|R_{a}\left(T_{s}\right)\right|+\left|R_{b}\left(T_{s}\right)\right|}{2\left|\rho\left(T_{s}\right)\right|},
$$

and $\rho_{h v}(0)$ can be computed by substituting (85), (86), and (91) into (76).

\section{Polarimetric Variables Measurement in STSR Mode}

STSR mode is based on the approximation that the propagation and backscattering matrices in $H / V$ polarization basis are diagonal. In this mode, radars with mechanically steered beams simultaneously transmit and receive both horizontal and vertical polarization states, and only reflectivity, differential reflectivity, differential phase, and copolar crosscorrelation coefficient can be estimated.

However, when the $L / M$ polarization basis is used, the propagation and backscattering matrices are not diagonal, and the four terms of $\mathbf{S}_{l m}^{\prime}$ cannot be measured separately at STSR mode. Assuming the same time index $(i)$ and with $s_{h v}^{\prime}=s_{v h}^{\prime}=0$, the simultaneously received baseband voltages in STSR mode can be derived from (26)

$$
\begin{aligned}
V_{l}^{r}(i)= & N_{\mathrm{or}}^{2}\left[s_{l l}^{\prime}(i) E_{t 1}+s_{l m}^{\prime}(i) E_{t 2}\right] \\
= & N_{\mathrm{or}}^{2}\left[a^{2} s_{h h}^{\prime}(i)+b^{2} s_{v v}^{\prime}(i)\right] E_{t 1} \\
& +N_{\mathrm{or}}^{2}\left[a b s_{h h}^{\prime}(i)-a b s_{v v}^{\prime}(i)\right] E_{t 2}, \\
V_{m}^{r}(i)= & N_{\mathrm{or}}^{2}\left[s_{m l}^{\prime}(i) E_{t 1}+s_{m m}^{\prime}(i) E_{t 2}\right] \\
= & N_{\mathrm{or}}^{2}\left[a b s_{h h}^{\prime}(i)-a b s_{v v}^{\prime}(i)\right] E_{t 1} \\
& +N_{\mathrm{or}}^{2}\left[b^{2} s_{h h}^{\prime}(i)+a^{2} s_{v v}^{\prime}(i)\right] E_{t 2} .
\end{aligned}
$$

$s_{h h}^{\prime}(i)$ and $s_{v v}^{\prime}(i)$ can be directly solved from the linear equations (92) and (93)

$$
\begin{aligned}
& s_{h h}^{\prime}(i)=\frac{a V_{l}^{r}(i)+b V_{m}^{r}(i)}{N_{\text {or }}^{2}\left(a E_{t 1}+b E_{t 2}\right)}, \\
& s_{v v}^{\prime}(i)=\frac{b V_{l}^{r}(i)-a V_{m}^{r}(i)}{N_{\text {or }}^{2}\left(b E_{t 1}-a E_{t 2}\right)} .
\end{aligned}
$$

So the simultaneous time series of $s_{h h}^{\prime}(i)$ and $s_{v v}^{\prime}(i)$ can be solved upon each return, and conventional polarimetric variables estimate methods of STSR mode can be used [2, ch6].

The more efficient procedure is using the following powers and correlations of received baseband voltages:

$P_{l}$

$$
\begin{aligned}
& =\frac{1}{N} \sum_{i=0}^{N-1}\left|V_{l}^{r}(i)\right|^{2} \\
& =N_{\mathrm{or}}^{4}\left\{\begin{array}{c}
a^{2}\left|a E_{t 1}+b E_{t 2}\right|^{2}\left\langle\left|s_{h h}^{\prime}\right|^{2}\right\rangle+b^{2}\left|b E_{t 1}-a E_{t 2}\right|^{2}\left\langle\left|s_{v v}^{\prime}\right|^{2}\right\rangle \\
+2 a b \operatorname{Re}\left[\left(a E_{t 1}+b E_{t 2}\right)^{*}\left(b E_{t 1}-a E_{t 2}\right)\left\langle s_{h h}^{\prime}{ }^{*} s_{v v}^{\prime}\right\rangle\right]
\end{array}\right\},
\end{aligned}
$$

$P_{m}$

$$
\begin{aligned}
& =\frac{1}{N} \sum_{i=0}^{N-1}\left|V_{m}^{r}(i)\right|^{2} \\
& =N_{\text {or }}^{4}\left\{\begin{array}{c}
b^{2}\left|a E_{t 1}+b E_{t 2}\right|^{2}\left\langle\left|s_{h h}^{\prime}\right|^{2}\right\rangle+a^{2}\left|-b E_{t 1}+a E_{t 2}\right|^{2}\left\langle\left|s_{v v}^{\prime}\right|^{2}\right\rangle \\
-2 a b \operatorname{Re}\left[\left(a E_{t 1}+b E_{t 2}\right)^{*}\left(b E_{t 1}-a E_{t 2}\right)\left\langle s_{h h}^{\prime} *(i) s_{v v}^{\prime}(i)\right\rangle\right]
\end{array}\right\},
\end{aligned}
$$

$R_{l, m}$

$=\frac{1}{N} \sum_{i=0}^{N-1} V_{l}^{r}(i) V_{m}^{r *}(i)$

$$
=N_{\mathrm{or}}^{4}\left\{\begin{array}{c}
a b\left|a E_{t 1}+b E_{t 2}\right|^{2}\left\langle\left|s_{h h}^{\prime}\right|^{2}\right\rangle-a b\left|b E_{t 1}-a E_{t 2}\right|^{2}\left\langle\left|s_{v v}^{\prime}\right|^{2}\right\rangle \\
-a^{2}\left(a E_{t 1}+b E_{t 2}\right)\left(b E_{t 1}-a E_{t 2}\right)^{*}\left\langle s_{h h^{\prime}}^{\prime} s_{v v}^{\prime}\right\rangle \\
+b^{2}\left(b E_{t 1}-a E_{t 2}\right)\left(a E_{t 1}+b E_{t 2}\right)^{*}\left\langle s_{h h}^{\prime}{ }^{*} s_{v v}^{\prime}\right\rangle
\end{array}\right\} .
$$

The three elements $\left\langle\left|s_{h h}^{\prime}\right|^{2}\right\rangle,\left\langle\left|s_{v v}^{\prime}\right|^{2}\right\rangle$, and $\left\langle s_{h h}^{\prime}{ }^{*} s_{v v}^{\prime}\right\rangle$ can be directly obtained from (95)-(97)

$$
\begin{gathered}
\left\langle\left|s_{h h}^{\prime}\right|^{2}\right\rangle=\frac{1}{N_{\text {or }}^{4}} \frac{a^{2} P_{l}+b^{2} P_{m}+2 a b \operatorname{Re}\left(R_{l, m}\right)}{\left|a E_{t 1}+b E_{t 2}\right|^{2}}, \\
\left\langle\left|s_{v v}^{\prime}\right|^{2}\right\rangle=\frac{1}{N_{\text {or }}^{4}} \frac{b^{2} P_{l}+a^{2} P_{m}-2 a b \operatorname{Re}\left(R_{l, m}\right)}{\left|b E_{t 1}-a E_{t 2}\right|^{2}}, \\
\left\langle s_{h h}^{\prime}{ }^{*} s_{v v}^{\prime}\right\rangle=\frac{1}{N_{\text {or }}^{4}} \frac{a b\left(P_{l}-P_{m}\right)-a^{2} R_{l, m}^{*}+b^{2} R_{l, m}}{\left(a E_{t 1}+b E_{t 2}\right)^{*}\left(b E_{t 1}-a E_{t 2}\right)} .
\end{gathered}
$$

As aforementioned, the magnitudes and phase differences of $E_{t 1}$ and $E_{t 1}$ need to be carefully measured and calibrated to successfully retrieve these covariance elements. The three polarimetric variables $Z_{\mathrm{DR}}, \phi_{\mathrm{DP}}$ and $\rho_{h v}(0)$ can then be computed by substituting (98), (99), and (100) into (72), (75), and (76), respectively.

\section{Summary and Conclusion}

This paper focuses on the accurate measurement of polarimetric variables in phased array weather radar. A new scheme 
of polarimetric measurement was proposed for the planar PPAR to mitigate the measurement biases in polarimetric variables caused by the misprojections of dual-polarized element's fields onto the local $H$ and $V$ directions of electronically steered beam. There are some differences between the proposed scheme and the projection correction scheme proposed in $[18,20]$. The basic ideal of [20] is based on simultaneously adjusting two polarized feeding voltages of array elements so that the $H$ and $V$ polarized fields in all beam directions are similar with that generated by mechanically steered antenna. The misprojection problem was considered in this paper by constructing a new polarization basis based on the orthogonal Huygens sources element model. The dual-polarized element's fields are symmetrically projected in the new polarization basis. Thereby, the copolar synthesized beam in the new basis can match well, and the measurement biases are only caused by the cross-polar radiations of element in new basis. Another benefit from the new basis is that each polarization can be synthesized individually, which would make polarized beam synthesizing process more flexible and straightforward.

The measured time series data in the new polarization basis need to be transformed to the conventional $H / V$ polarization basis so that polarimetric variables can be estimated. Both the ATSR and the STSR modes were considered, and two methods of estimating polarimetric variables have been developed. The first way is to transform the measured scattering matrix in new basis to the scattering matrix in $H / V$ basis. The scattering conversion matrix and polarimetric variables can then be computed by using the same procedure as radars with mechanically steered antenna. Another way is to directly compute the powers and correlation of measured data, and polarimetric variables can then be estimated with the developed formulas.

The basic theories and methods of polarimetric measurement in the new polarization basis have been developed. Further practical issues such as bias tolerances caused by attentions in propagation path and by the canting of drops need to be analyzed, and the tolerable cross-polarization in the new basis for reliable precipitation estimation also needs to be reinvestigated. The ongoing work in our team is to develop an $8 \times 8$ small array comprising dual-polarized balance feed microstrip elements, which will be used for further validation.

\section{Acknowledgments}

This work was supported by the National Natural Science Foundation of China (CSNF) under Grant 61201330. The authors sincerely express their gratitude to the anonymous reviewers and the editors for their helpful and constructive suggestions.

\section{References}

[1] R. J. Doviak and D. S. Zrnic, Doppler Radar and Weather Observations, Academic Press, New York, NY, USA, 2nd edition, 1993.
[2] V. N. Bringi and V. Chandrasekar, Polarimetric Doppler Weather Radar, Cambridge University Press, Cambridge, UK, 2001.

[3] R. J. Doviak, V. Bringi, A. Ryzhkov, A. Zahrai, and D. Zrnić, "Considerations for polarimetric upgrades to operational WSR-88D radars," Journal of Atmospheric and Oceanic Technology, vol. 17, no. 3, pp. 257-278, 2000.

[4] R. J. Doviak and D. S. Zrnic, WSR-88D Radar for Research and Enhancement of Operations: Polarimetric Upgrades to Improve Rainfall Measurements, Norman, OK: NOAA/NSSL, 1998, http://publications.nssl.noaa.gov/wsr88d_reports/2pol_ upgrades.pdf.

[5] V. Chandrasekar, J. Hubbert, V. N. Bringi, and P. F. Meischner, "Analysis and interpretation of dual-polarized radar measurements at +45 and -45 linear polarization states," Journal of Atmospheric \& Oceanic Technology, vol. 11, no. 2, pp. 323-336, 1994.

[6] V. Chandrasekar, J. Hubert, V. N. Bringi, and P. F. Meischner, "Transformation of dual-polarized radar measurements to arbitrary polarization bases," Journal of Atmospheric \& Oceanic Technology, vol. 11, no. 4, pp. 937-949, 1994.

[7] N. Bharadwaj and V. Chandrasekar, "Phase coding for range ambiguity mitigation in dual-polarized Doppler weather radars," Journal of Atmospheric and Oceanic Technology, vol. 24, no. 8, pp. 1351-1363, 2007.

[8] V. Chandrasekar and N. Bharadwaj, "Orthogonal channel coding for simultaneous co- and cross-polarization measurements," Journal of Atmospheric and Oceanic Technology, vol. 26, no. 1, pp. 4556-1363, 2009.

[9] V. Chandrasekar and R. J. Keeler, "Antenna pattern analysis and measurements for multiparameter radars," Journal of Atmospheric \& Oceanic Technology, vol. 10, no. 5, pp. 674-683, 1993.

[10] Y. Wang and V. Chandrasekar, "Polarization isolation requirements for linear dual-polarization weather radar in simultaneous transmission mode of operation," IEEE Transactions on Geoscience and Remote Sensing, vol. 44, no. 8, pp. 2019-2028, 2006.

[11] D. Zrnić, R. Doviak, G. Zhang, and A. Ryzhkov, "Bias in differential reflectivity due to cross coupling through the radiation patterns of polarimetric weather radars," Journal of Atmospheric and Oceanic Technology, vol. 27, no. 10, pp. 1624-1637, 2010.

[12] M. Galletti and D. S. Zrnic, "Bias incopolar correlation coefficient caused by antenna radiation patterns," IEEE Transactions on Geoscience and Remote Sensing, vol. 49, no. 6, pp. 22742280, 2011.

[13] M. E. Weber, J. Y. N. Cho, J. S. Herd, J. M. Flavin, W. E. Benner, and G. S. Torok, "The next-generation multimission U.S. Surveillance radar network," Bulletin of the American Meteorological Society, vol. 88, no. 11, pp. 1739-1751, 2007.

[14] D. S. Zrnic, J. F. Kimpel, D. E. Forsyth et al., "Agile-beam phased array radar for weather observations," Bulletin of the American Meteorological Society, vol. 88, no. 11, pp. 17531766, 2007.

[15] P. L. Heinselman, D. L. Priegnitz, K. L. Manross, T. M. Smith, and R. W. Adams, "Rapid sampling of severe storms by the National Weather Radar Testbed Phased Array Radar," Weather and Forecasting, vol. 23, no. 5, pp. 808-824, 2008.

[16] G. Zhang, R. Doviak, D. Zrnic, and J. Crain, "Phased array radar polarimetry for weather sensing: challenges and opportunities," in Proceedings of the IEEE International Geoscience and Remote Sensing Symposium (IGARSS '08), pp. V449V452, Boston, Mass, USA, July 2008. 
[17] A. O. Boryssenko, "Polarization constraints in dual-polarized phased arrays derived from an infinite current sheet model," IEEE Antennas and Wireless Propagation Letters, vol. 8, pp. 955-958, 2009.

[18] G. Zhang, R. J. Doviak, D. S. Zrnic, J. Crain, D. Staiman, and Y. Al-Rashid, "Phased array radar polarimetry for weather sensing: a theoretical formulation for bias corrections," IEEE Transactions on Geoscience and Remote Sensing, vol. 47, no. 11, pp. 3679-3689, 2009.

[19] R. J. Doviak, L. Lei, G. Zhang, J. Meier, and C. Curtis, "Comparing theory and measurements of cross-polar fields of a phased-array weather radar," IEEE Geoscience and Remote Sensing Letters, vol. 8, no. 5, pp. 1002-1006, 2011.

[20] D. S. Zrnic, G. Zhang, and R. J. Doviak, "Bias correction and doppler measurement for polarimetric phased-array radar," IEEE Transactions on Geoscience and Remote Sensing, vol. 49, no. 2, pp. 843-853, 2011.

[21] G. Zhang, R. J. Doviak, D. S. Zrnić, R. Palmer, L. Lei, and Y. Al-Rashid, "Polarimetric phased-array radar for weather measurement: a planar or cylindrical configuration?" Journal of Atmospheric and Oceanic Technology, vol. 28, no. 1, pp. 63-73, 2011.

[22] M. S. Barbetty, R. W. Jackson, and S. Frasier, "Interleaved sparse arrays for polarization control of electronically steered phased arrays for meteorological applications," IEEE Transactions on Geoscience and Remote Sensing, vol. 50, no. 4, pp. 1283-1290, 2012.

[23] D. Staiman, "Calibration of polarimetric phased array radar for impoved measurement accuracy," in Proceedings of the 25th International Conference on IIPS for Meteorology, Oceanography, and Hydrology, January 2009.

[24] C. Fulton and W. J. Chappell, "Calibration of a digital phased array for polarimetric radar," in Proceedings of the IEEE MTT-S International Microwave Symposium (MTT '10), pp. 161-164, May 2010.

[25] C. Fulton and W. Chappell, "Calibration of panelized polarimetric phased array radar antennas: a case study," in Proceedings of the 4th IEEE International Symposium on Phased Array Systems and Technology (Array '10), pp. 860-867, October 2010.

[26] A. W. Love, "Some highlights in reflector antenna development," Radio Science, vol. 11, no. 8-9, pp. 671-684, 1976.

[27] P. Alitalo, A. O. Karilainen, T. Niemi, C. R. Simovski, and S. A. Tretyakov, "Design and realisation of an electrically small Huygens source for circular polarisation," IET Microwaves, Antennas and Propagation, vol. 5, no. 7, pp. 783-789, 2011.

[28] C. A. Balanis, Antenna Theory Analysis and Design, John Wiley \& Sons, Hoboken, NJ, USA, 3rd edition, 2005.

[29] P. Jin and R. W. Ziolkowski, "Metamaterial-inspired, electrically small huygens sources," IEEE Antennas and Wireless Propagation Letters, vol. 9, pp. 501-505, 2010.

[30] C. Fulton and W. J. Chappell, "A Dual-Polarized patch antenna for weather radar applications," in IEEE International Symposium on Microwaves, Communications, Antennas and Electronics Systems, November 2011.

[31] A. Ludwig, "The definition of cross polarization," IEEE Transactions on Antennas and Propagation, vol. 21, no. 1, pp. 116119, 1973.

[32] S. Gregson, J. McCormick, and C. Parini, Principles of Planar Near-Field Antenna Measurements, The Institution of Engineering and Technology, London, UK, 2007.

[33] D. F. Kelley and W. L. Stutzman, "Array antenna pattern modeling methods that include mutual coupling effects," IEEE
Transactions on Antennas and Propagation, vol. 41, no. 12, pp. 1625-1632, 1993.

[34] K. Tragl, "Polarimetric radar backscattering from reciprocal random targets," IEEE Transactions on Geoscience and Remote Sensing, vol. 28, no. 5, pp. 856-864, 1990.

[35] D. S. Zrnic, "Complete polarimetric measurements with a single receiver radar," Journal of Atmospheric and Oceanic Technology, vol. 8, no. 1, pp. 159-165, 1991.

[36] M. Sachidananda and D. S. Zrnic, "ZDR measurement considerations for a fast scan capability radar," Radio Science, vol. 20, pp. 907-922, 1985.

[37] M. Sachidananda and D. S. Zrnic, "Efficient processing of alternately polarization radar echos," Journal of Atmospheric and Oceanic Technology, vol. 4, no. 4, pp. 1310-1318, 1988. 

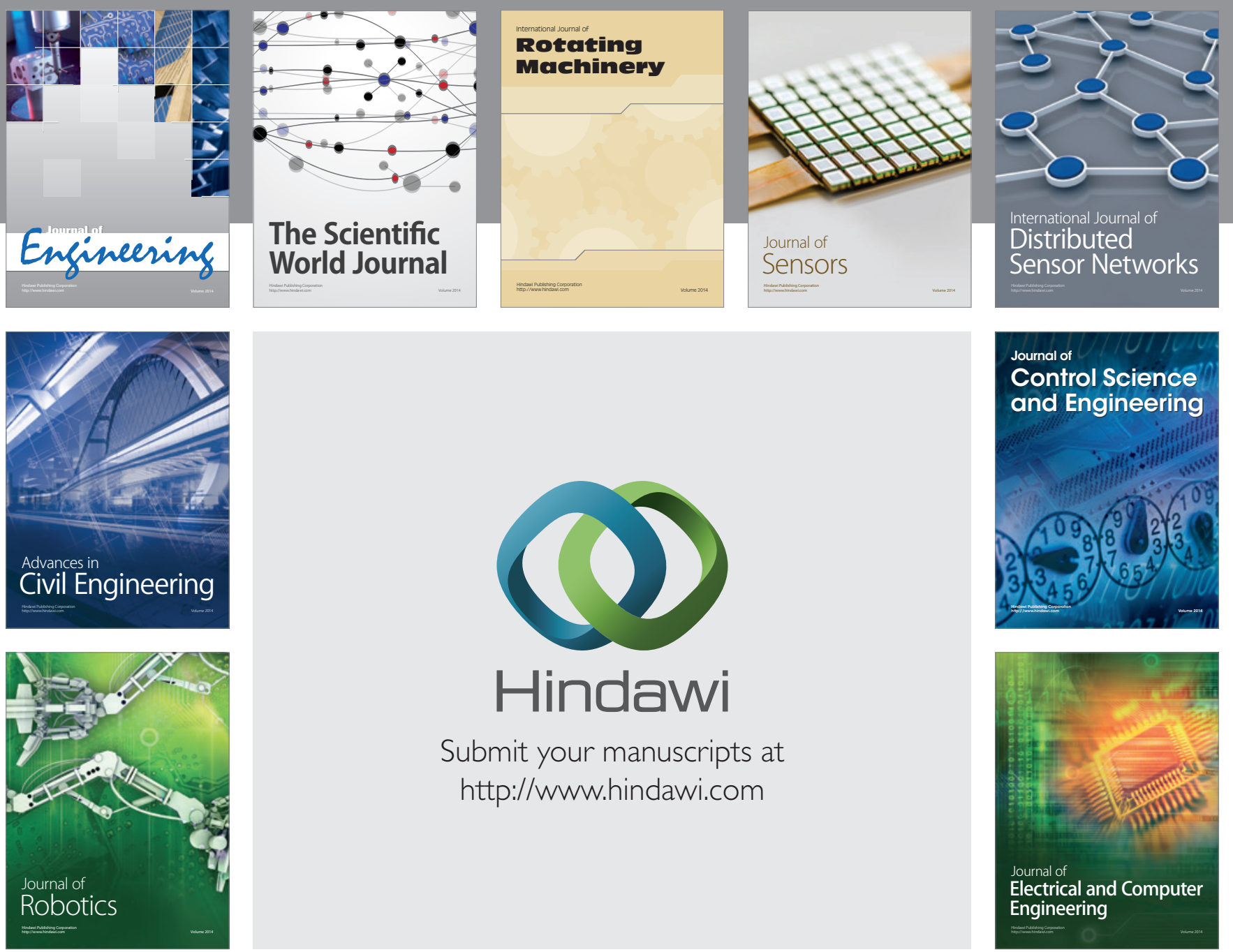

Submit your manuscripts at

http://www.hindawi.com
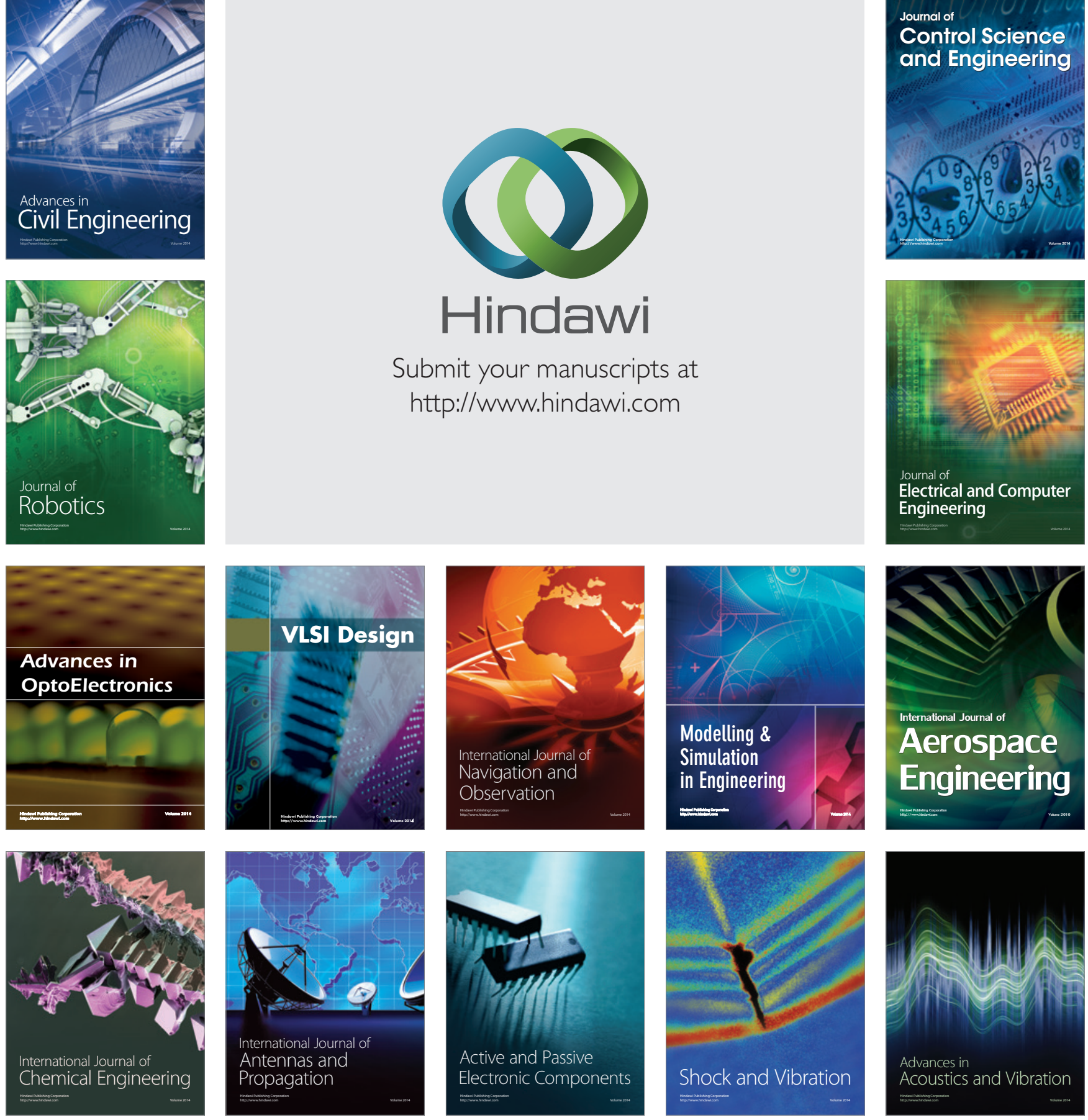